Available online at GSC Online Press Directory

GSC Advanced Research and Reviews e-ISSN: 2582-4597, CODEN (USA): GARRC2

Journal homepage: https://www.gsconlinepress.com/journals/gscarr

(RESEARCH ARTICLE)

\title{
On a curve and a system
}

\author{
Tuba Ağırman Aydın 1,*, Seda Çayan ${ }^{2}$, Mehmet Sezer ${ }^{3}$ and Abdullah Mağden 4 \\ ${ }^{1}$ Faculty of Education, Bayburt University, Bayburt, Turkey. \\ ${ }^{2}$ Faculty of Science and Arts, Manisa Celal Bayar University, Manisa, Turkey. \\ ${ }^{3}$ Faculty of Science and Arts, Manisa Celal Bayar University, Manisa, Turkey. \\ ${ }^{4}$ Faculty of Engineering and Natural Sciences, Bursa Technical University, Bursa, Turkey.
}

Publication history: Received on 10 November 2020; revised on 17 November 2020; accepted on 19 November 2020

Article DOI: https://doi.org/10.30574/gscarr.2020.5.3.0101

\begin{abstract}
Curves of constant width, which have a very special place in many fields such as kinematics, engineering, art, cam design and geometry, are specially discussed under this title. In this study, a system of differential equations characterizing the curves of constant width is examined. This is the system of the first order homogenous differential equations with variable coefficients in the normal form. Approximate solutions of the system, by means of two different polynomial approaches, are calculated and error analysis is made. The obtained results are analyzed on a numerical sample and the best method of approach is determined. This system can also constitute a characterization for different types of curves according to different frames in different spaces. Therefore, this study is important not only for this curve type but also for the geometry of all curves that can be expressed in a similar system.
\end{abstract}

Keywords: Curves of Constant Width; Hermite Matrix Collocation Method; Taylor Matrix Collocation Method; Frenet Frame; Reuleaux Triangle

\section{Introduction}

Two points on a curve where the tangents are parallel, and opposite are called opposing points. The distance between these two points is expressed as the width of the curve. A pair of support lines that are parallel to each other, perpendicular to the given direction, the distance between them is defined as the width of a figure in the chosen direction. The curve whose distance between all opposing points on it is constant and equal to each other is defined as the curve of constant width. Also, shapes that have the same width in each direction are called shapes of constant width $[1,2]$.

These particular curves and shapes, along with some of their properties, have been known for a long time. Firstly, Euler studies them under the name Latin orbiforms for circular curves. He is interested in curves of constant width whose boundaries can be represented as the evolution of a hypocycloid. Later, Reuleaux, an engineer and mathematician, also considered shapes of constant width in his book on kinematics in engineering. In this book, he describes the triangle, which is probably the simplest shape of constant width after the circle and bears his name today. This geometric shape, the Reuleaux triangle, has been known for a long time, but Reuleaux is the first person to focus on constant width properties $[3,4]$. The Reuleaux triangle is obtained starting from the equilateral triangle. It is possible to create shapes of constant width on different regular polygons with similar logic.

\footnotetext{
${ }^{*}$ Corresponding author: Tuba AĞIRMAN AYDIN

Faculty of Education, Bayburt University, Bayburt, Turkey.

Copyright (C) 2020 Author(s) retain the copyright of this article. This article is published under the terms of the Creative Commons Attribution Liscense 4.0.
} 
The shapes of constant width are convex shapes that don't pass through their own surface area. Many geometers have shown interest in this type of curve because of the properties that make them different $[5,6,7,8]$. It is possible to observe the use of shapes with this characterization in many branches of science. These curves have many different applications in engineering such as cams (shaft bends, machine part), square hole drilling drills and manhole covers, pistons of rotary, internal combustion engines. A part of a simple cam mechanism used in most older cinema projectors is a special curve of constant width $[2,9]$. Water wheels are built on the system that contains this type of curve. The basic curves used in the localization and coverage areas of wireless networks are of this type $[10,11,12]$. There are many decorative applications in art where constant width curve figures play an important role. Especially the most widely known shape of constant width, the circle or circular cross-section cylinder is used in many mechanisms, architecture and design. What makes the circle and the circular part more preferable is that both are accessible in terms of equation and application. However, it has been observed in different study areas that the use of different curves with fixed widths outside the circle has some advantages. For example, it has been shown by calculations that the probability of overturning of a road tanker with a side section in the form of the Reuleaux triangle is less than that of a tanker whose side section is in the form of a circle [13]. Examples like this can be multiplied. Here, the reason why a more advantageous situation is not preferred can be shown as the insufficient information about the alternative. In fact, it is possible to find many studies to close this gap $[14,15]$.

In this article, first order, homogeneous, linear differential equation system with variable coefficients that characterizes curves of constant width is obtained. Approximate solutions of this system are calculated by two different methods and error analysis is made. The most suitable approximation method for these curves is determined with the help of error analysis on a numerical sample. The expression of the curve of constant width with differential equations will prepare the ground for the comfortable use of these curves in every field. In addition, the opportunity to experience different models under the same title will provide higher efficiency in use.

\section{Material and methods}

\subsection{The space curves of constant width}

Let the curve $\gamma$ be a simple closed curve with unit speed from class $C^{3}$ with non-zero curvature and torsion in Euclid space. Let $\gamma(s)$ and $\gamma^{*}(s)$ be two opposite points on the curve. The vector position of the curve $\gamma$ is expressed in

$$
\gamma^{*}(s)=\gamma(s)+\lambda(s) \vec{t}+\mu(s) \vec{n}+\delta(s) \vec{b}
$$

where $\vec{t}, \vec{n}, \vec{b}$ are Frenet frame components. This equality is differentiated according to the arc length parameter s and

$$
\frac{d \gamma^{*}}{d s}=\vec{t}^{*} \frac{d s^{*}}{d s}=\left(1+\frac{d \lambda}{d s}-\mu(s) \kappa\right) \vec{t}+\left(\lambda(s) \kappa+\frac{d \mu}{d s}-\delta(s) \tau\right) \vec{n}+\left(\frac{d \delta}{d s}+\mu(s) \tau\right) \vec{b}
$$

expression is obtained by using Frenet's formulas. Since there is $\vec{t}=-\vec{t}^{*}$ at opposite points, the system

$$
\begin{aligned}
& -\frac{d s^{*}}{d s}=1+\frac{d \lambda}{d s}-\mu(s) \kappa \\
& \lambda(s) \kappa+\frac{d \mu}{d s}-\delta(s) \tau=0 \\
& \frac{d \delta}{d s}+\mu(s) \tau=0
\end{aligned}
$$

is found. It is known that the curvature of the curve $\gamma$ is $\kappa=\lim \frac{\Delta \varphi}{\Delta s}=\frac{d \varphi}{d s}$, with the contengency angle $\Delta \varphi$. Thus, the system of differential equations (2) can be written in the normal form 


$$
\begin{aligned}
& \frac{d \lambda}{d \varphi}=\mu-f(\varphi) \\
& \frac{d \mu}{d \varphi}=\rho \tau \delta-\lambda \\
& \frac{d \delta}{d \varphi}=-\rho \tau \mu .
\end{aligned}
$$

$\rho=\frac{1}{\kappa}, \rho^{*}=\frac{1}{\kappa^{*}}$ are the radii of curvature at the points $\gamma(s)$ and $\gamma^{*}(s)$, respectively and $f(\varphi)=\rho+\rho^{*}$. On the other hand, since the distance between the opposite points is constant, $\left\|\gamma^{*}-\gamma\right\|^{2}=\|d\|^{2}=\lambda^{2}+\mu^{2}+\delta^{2}=$ constant is written. If the equality $\lambda^{2}+\mu^{2}+\delta^{2}=$ constant is differentiated according to $\varphi, \lambda \lambda^{\prime}+\mu \mu^{\prime}+\delta \delta^{\prime}=0$ is obtained. This equality can be reduced to equality $\lambda\left(\lambda^{\prime}-\mu\right)=0$ with the help of the system (3). Two basic situations are possible here:

Case I: $\lambda=0$,

Case II: $\lambda^{\prime}-\mu=0$.

In the case I, $\mu=f(\varphi)$ is obtained from the equality (3.1). For $\lambda=0$ and $\mu=f(\varphi)$, the system of differential equations (3) becomes

$$
\begin{aligned}
& \mu=f(\varphi) \\
& \frac{d \mu}{d \varphi}=\rho \tau \delta \\
& \frac{d \delta}{d \varphi}=-\rho \tau \mu .
\end{aligned}
$$

From the second order linear equation with variable coefficient depending on the coefficient $\mu$ obtained by eliminating $\delta$ and its derivatives,

$$
\mu=A \cos \int_{0}^{\varphi} \rho \tau d \varphi+B \sin \int_{0}^{\varphi} \rho \tau d \varphi=f(\varphi)
$$

is obtained, with A and B being the integration constants. From the equation obtained by eliminating $\mu$ and its derivatives, $\delta$ is calculated as follows:

$$
\delta=-A \sin \int_{0}^{\varphi} \rho \tau d \varphi+B \cos \int_{0}^{\varphi} \rho \tau d \varphi
$$

If these equations are used in (1), the integral characterization of the curve for $\lambda=0$ would be as follows:

$$
\gamma^{*}=\gamma+\left[A \cos \int_{0}^{\varphi} \rho \tau d \varphi+B \sin \int_{0}^{\varphi} \rho \tau d \varphi\right] \vec{n}+\left[-A \sin \int_{0}^{\varphi} \rho \tau d \varphi+B \cos \int_{0}^{\varphi} \rho \tau d \varphi\right] \vec{b} .
$$


Also, in this case, the constant width value of the curve, i.e. the distance between the opposite points, is obtained as $\|d\|=\sqrt{A^{2}+B^{2}}$.

In the case II, $f=0$ is obtained from the equality (3.1). Since the distance $d$ will be constant for $f=0$, the curve $\gamma$ has a constant width. So, if $f=0$ is taken in the system (3), the following system of differential equations characterizing curves of constant width is obtained:

$$
\begin{aligned}
& \frac{d \lambda}{d \varphi}=\mu \\
& \frac{d \mu}{d \varphi}=\rho \tau \delta-\lambda \\
& \frac{d \delta}{d \varphi}=-\rho \tau \mu
\end{aligned}
$$

\subsection{Two solutions for the system representing the curve}

Let the approximate solutions of the differential equation system (4) by the Taylor matrix collocation method under the initial conditions

$$
\lambda(0)=\lambda_{0}, \quad \mu(0)=\mu_{0}, \quad \delta(0)=\delta_{0}
$$

in the interval $0 \leq \varphi \leq \chi$ be in the form of the truncated Taylor series

$$
\begin{aligned}
& \lambda \cong \lambda_{N}(\varphi)=\sum_{q=0}^{N} a_{1 q} \varphi^{q} \\
& \mu \cong \mu_{N}(\varphi)=\sum_{q=0}^{N} a_{2 q} \varphi^{q} \\
& \delta \cong \delta_{N}(\varphi)=\sum_{q=0}^{N} a_{3 q} \varphi^{q}
\end{aligned}
$$

and the approximate solutions obtained by the Hermite matrix collocation method be in the form of the truncated Hermite series

$$
\begin{aligned}
& \lambda \cong \tilde{\lambda}_{N}(\varphi)=\sum_{r=0}^{N} a_{1 r} H_{r}(\varphi) \\
& \mu \cong \tilde{\mu}_{N}(\varphi)=\sum_{r=0}^{N} a_{2 r} H_{r}(\varphi) \\
& \delta \cong \tilde{\delta}_{N}(\varphi)=\sum_{r=0}^{N} a_{3 r} H_{r}(\varphi)
\end{aligned}
$$

$a_{i q}(i=1,2,3)$ are the unknown coefficients of the Taylor series, $a_{j r} \quad(j=1,2,3)$ are unknown Hermite coefficients,

$$
H_{r}(\varphi)=\sum_{k=0}^{[r / 2]}(-1)^{k} \frac{r !}{(r-2 k) ! k !} 2^{r-2 k} \varphi^{r-2 k}
$$


are Hermite polynomials and $N$ is any positive integer with the truncation limit $N \geq 1$ [14].

In order to find the approximate solutions of the differential equation system (4) under the initial conditions (5), firstly, the system (4) is written as

$$
\underbrace{\left[\begin{array}{lll}
1 & 0 & 0 \\
0 & 1 & 0 \\
0 & 0 & 1
\end{array}\right]}_{\mathbf{P}_{1}(\varphi)}\left[\begin{array}{l}
\lambda^{\prime} \\
\mu^{\prime} \\
\delta^{\prime}
\end{array}\right]+\underbrace{\left[\begin{array}{ccc}
0 & -1 & 0 \\
1 & 0 & -\rho \tau \\
0 & \rho \tau & 0
\end{array}\right]}_{\mathbf{P}_{0}(\varphi)} \quad\left[\begin{array}{l}
\lambda \\
\mu \\
\delta
\end{array}\right]=\left[\begin{array}{l}
0 \\
0 \\
0
\end{array}\right]
$$

in matrix form and the matrix relation

$$
\mathbf{P}_{1}(\varphi) \boldsymbol{\Lambda}^{\prime}(\varphi)+\mathbf{P}_{0}(\varphi) \boldsymbol{\Lambda}(\varphi)=\mathbf{G}(\varphi)
$$

is obtained. Then, in order to obtain approximate solutions with Taylor matrix collocation method, the equations (6.1), (6.2) and (6.3) are written in the matrix forms as follows:

$\lambda_{N}(\varphi)=\sum_{q=0}^{N} a_{1 q} \varphi^{q}=\underbrace{\left[\begin{array}{cccc}1 & \varphi & \cdots & \varphi^{N}\end{array}\right]}_{\mathbf{X}(\varphi)}\left[\begin{array}{c}a_{10} \\ a_{11} \\ \vdots \\ a_{1 N}\end{array}\right]=\mathbf{X}(\varphi) \mathbf{A}_{1}$,

$\mathbf{A}_{1}$

$\mu_{N}(\varphi)=\sum_{q=0}^{N} a_{2 q} \varphi^{q}=\underbrace{\left[\begin{array}{cccc}1 & \varphi & \cdots & \varphi^{N}\end{array}\right]}_{\mathbf{X}(\varphi)}\left[\begin{array}{c}a_{20} \\ a_{21} \\ \vdots \\ a_{2 N}\end{array}\right]=\mathbf{X}(\varphi) \mathbf{A}_{2}$,

$\mathbf{A}_{2}$

$\delta_{N}(\varphi)=\sum_{q=0}^{N} a_{3 q} \varphi^{q}=\underbrace{\left[\begin{array}{cccc}1 & \varphi & \cdots & \varphi^{N}\end{array}\right]}_{\mathbf{X}(\varphi)}\left[\begin{array}{c}a_{30} \\ a_{31} \\ \vdots \\ a_{3 N}\end{array}\right]=\mathbf{X}(\varphi) \mathbf{A}_{3}$

$\mathbf{A}_{3}$

or in short

$$
\begin{aligned}
\lambda_{N}(\varphi) & =\mathbf{X}(\varphi) \mathbf{A}_{1}, \\
\mu_{N}(\varphi) & =\mathbf{X}(\varphi) \mathbf{A}_{2}, \\
\delta_{N}(\varphi) & =\mathbf{X}(\varphi) \mathbf{A}_{3} .
\end{aligned}
$$

Then, the expression (10) is arranged and matrix relation 


$$
\underbrace{\left[\begin{array}{c}
\lambda_{N}(\varphi) \\
\mu_{N}(\varphi) \\
\delta_{N}(\varphi)
\end{array}\right]}_{\boldsymbol{\Lambda}(\varphi)}=\underbrace{\left[\begin{array}{ccc}
\mathbf{X}(\varphi) & 0 & 0 \\
0 & \mathbf{X}(\varphi) & 0 \\
0 & 0 & \mathbf{X}(\varphi)
\end{array}\right]}_{\overline{\mathbf{X}}(\varphi)} \underbrace{\mathbf{A}_{3}}_{\mathbf{A}}] \begin{aligned}
& \mathbf{A}_{1} \\
& \mathbf{A}_{2} \\
& \mathbf{A}_{3}
\end{aligned} \Rightarrow \boldsymbol{\Lambda}(\varphi)=\overline{\mathbf{X}}(\varphi) \mathbf{A}
$$

is obtained. On the other hand, the relation between matrices $\mathbf{X}(\varphi)$ and $\mathbf{X}^{\prime}(\varphi)$ is

$$
\mathbf{X}^{\prime}(\varphi)=\mathbf{X}(\varphi) \mathbf{B} ; \quad \mathbf{B}=\left[\begin{array}{ccccc}
0 & 1 & 0 & \cdots & 0 \\
0 & 0 & 2 & \cdots & 0 \\
\vdots & \vdots & \vdots & \ddots & \vdots \\
0 & 0 & 0 & \cdots & N \\
0 & 0 & 0 & \cdots & 0
\end{array}\right]
$$

thus, the equalities

$$
\begin{aligned}
& \lambda_{N}^{\prime}(\varphi)=\mathbf{X}^{\prime}(\varphi) \mathbf{A}_{1}=\mathbf{X}(\varphi) \mathbf{B} \mathbf{A}_{1}, \\
& \mu_{N}^{\prime}(\varphi)=\mathbf{X}^{\prime}(\varphi) \mathbf{A}_{2}=\mathbf{X}(\varphi) \mathbf{B} \mathbf{A}_{2}, \\
& \delta_{N}^{\prime}(\varphi)=\mathbf{X}^{\prime}(\varphi) \mathbf{A}_{3}=\mathbf{X}(\varphi) \mathbf{B} \mathbf{A}_{3} .
\end{aligned}
$$

are written. The expression (13) is arranged and

$$
\underbrace{\left[\begin{array}{c}
\lambda_{N}^{\prime}(\varphi) \\
\mu_{N}^{\prime}(\varphi) \\
\delta_{N}^{\prime}(\varphi)
\end{array}\right]}_{\boldsymbol{\Lambda}^{\prime}(\varphi)}=\underbrace{\left[\begin{array}{ccc}
\mathbf{X}(\varphi) & 0 & 0 \\
0 & \mathbf{X}(\varphi) & 0 \\
0 & 0 & \mathbf{X}(\varphi)
\end{array}\right]}_{\overline{\mathbf{X}}(\varphi)} \underbrace{\left[\begin{array}{lll}
\mathbf{B} & 0 & 0 \\
0 & \mathbf{B} & 0 \\
0 & 0 & \mathbf{B}
\end{array}\right]}_{\mathbf{B}}\left[\begin{array}{l}
\mathbf{A}_{1} \\
\mathbf{A}_{2} \\
\mathbf{A}_{3}
\end{array}\right] \Rightarrow \mathbf{\Lambda}^{\prime}(\varphi)=\overline{\mathbf{X}}(\varphi) \overline{\mathbf{B}} \mathbf{A}
$$

is obtained in matrix form. By writing the equations (11) and (14) into the equation (9), the matrix relation

$$
\mathbf{P}_{1}(\varphi) \overline{\mathbf{X}}(\varphi) \overline{\mathbf{B}} \mathbf{A}+\mathbf{P}_{0}(\varphi) \overline{\mathbf{X}}(\varphi) \mathbf{A}=\mathbf{G}(\varphi)
$$

is found. Then, by writing the collocation points defined with

$$
\varphi_{\xi}=\frac{\chi}{N} \xi, \quad \xi=0,1,2, \ldots, N
$$

into their place in the matrix relation (15), the fundamental matrix relation

$$
\left\{\mathbf{P}_{1} \overline{\mathbf{X}} \overline{\mathbf{B}}^{*}+\mathbf{P}_{0} \overline{\mathbf{X}} \overline{\mathbf{I}}^{*}\right\} \mathbf{A}=\mathbf{G}
$$

is obtained. The matrices here are as follows: 


$$
\begin{aligned}
& \mathbf{P}_{1}=\left[\begin{array}{cccc}
\mathbf{P}_{1}\left(\varphi_{0}\right) & 0 & \cdots & 0 \\
0 & \mathbf{P}_{1}\left(\varphi_{1}\right) & \cdots & 0 \\
\vdots & \vdots & \ddots & \vdots \\
0 & 0 & \cdots & \mathbf{P}_{1}\left(\varphi_{N}\right)
\end{array}\right], \mathbf{P}_{0}=\left[\begin{array}{cccc}
\mathbf{P}_{0}\left(\varphi_{0}\right) & 0 & \cdots & 0 \\
0 & \mathbf{P}_{0}\left(\varphi_{1}\right) & \cdots & 0 \\
\vdots & \vdots & \ddots & \vdots \\
0 & 0 & \cdots & \mathbf{P}_{0}\left(\varphi_{N}\right)
\end{array}\right], \overline{\mathbf{B}}^{*}=\left[\begin{array}{c}
\overline{\mathbf{B}} \\
\overline{\mathbf{B}} \\
\vdots \\
\overline{\mathbf{B}}
\end{array}\right] \\
& \overline{\mathbf{X}}=\left[\begin{array}{cccc}
\overline{\mathbf{X}}\left(\varphi_{0}\right) & 0 & \cdots & 0 \\
0 & \overline{\mathbf{X}}\left(\varphi_{1}\right) & \cdots & 0 \\
\vdots & \vdots & \ddots & \vdots \\
0 & 0 & \cdots & \overline{\mathbf{X}}\left(\varphi_{N}\right)
\end{array}\right], \overline{\mathbf{I}}^{*}=\left[\begin{array}{c}
\overline{\mathbf{I}} \\
\overline{\mathbf{I}} \\
\vdots \\
\overline{\mathbf{I}}
\end{array}\right], \quad \overline{\mathbf{I}}=\left[\begin{array}{cccc}
\mathbf{I} & 0 & \cdots & 0 \\
0 & \mathbf{I} & \cdots & 0 \\
\vdots & \vdots & \ddots & \vdots \\
0 & 0 & \cdots & \mathbf{I}
\end{array}\right], \\
& \mathbf{I}=\left[\begin{array}{cccc}
1 & 0 & \cdots & 0 \\
0 & 1 & \cdots & 0 \\
\vdots & \vdots & \ddots & \vdots \\
0 & 0 & \cdots & 1
\end{array}\right], \quad \mathbf{G}=\left[\begin{array}{c}
\mathbf{G}\left(\varphi_{0}\right) \\
\mathbf{G}\left(\varphi_{1}\right) \\
\vdots \\
\mathbf{G}\left(\varphi_{N}\right)
\end{array}\right]=\left[\begin{array}{c}
\mathbf{0} \\
\mathbf{0} \\
\vdots \\
\mathbf{0}
\end{array}\right]
\end{aligned}
$$

The fundamental matrix equation (17) can be written in short form as

$$
\mathbf{W A}=\mathbf{G} \text { or }[\mathbf{W} ; \mathbf{G}] ; \quad \mathbf{W}=\mathbf{P}_{1} \overline{\mathbf{X}} \overline{\mathbf{B}}^{*}+\mathbf{P}_{0} \overline{\mathbf{X}} \overline{\mathbf{I}}^{*}
$$

On the other hand, the equations (10) can be expressed as follows by substituting in the initial conditions (5):

$$
\begin{aligned}
& \lambda(0)=\lambda_{0} \Rightarrow \mathbf{X}(0) \mathbf{A}_{1}=\lambda_{0}, \\
& \mu(0)=\mu_{0} \Rightarrow \mathbf{X}(0) \mathbf{A}_{2}=\mu_{0}, \\
& \delta(0)=\delta_{0} \Rightarrow \mathbf{X}(0) \mathbf{A}_{3}=\delta_{0} .
\end{aligned}
$$

The matrix relations of conditions are obtained as

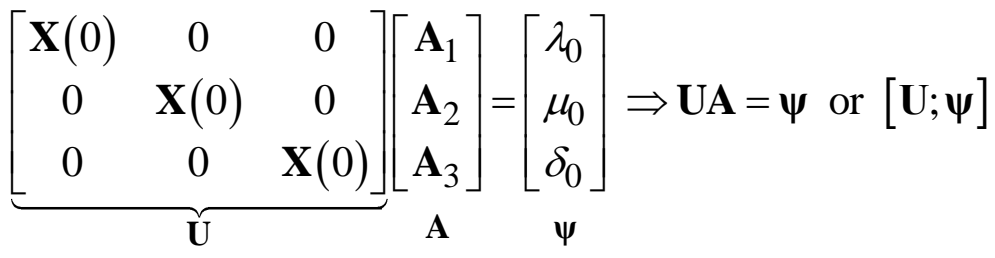

by arranging the expression (17). In order to obtain the solutions of the differential equation system (4) under initial conditions (5), the augmented matrix $[\tilde{\mathbf{W}} ; \tilde{\mathbf{G}}]$ is obtained by deleting any three rows of the augmented matrix $[\mathbf{W} ; \mathbf{G}]$ and writing the augmented matrix $[\mathbf{U} ; \boldsymbol{\psi}]$ of the conditions instead. If $\operatorname{rank}(\tilde{\mathbf{W}})=\operatorname{rank}(\tilde{\mathbf{W}} ; \tilde{\mathbf{G}})=3(N+1)$, the solution of augmented matrix $[\tilde{\mathbf{W}} ; \tilde{\mathbf{G}}]$ is obtained in the form $\mathbf{A}=(\tilde{\mathbf{W}})^{-1} \tilde{\mathbf{G}}$. Thus, the unknown coefficients of the Taylor series are obtained, and approximate solutions are found in the form of the truncated Taylor series (6). 
If specially, the interval $0 \leq \varphi \leq 2 \pi$ and truncation limit $N=3$ are selected; the collocation points are $\varphi_{0}=0, \varphi_{1}=\frac{2 \pi}{3}, \varphi_{2}=\frac{4 \pi}{3}, \varphi_{3}=2 \pi$ and matrices are

$\mathbf{X}(\varphi)=\left[\begin{array}{llll}1 & \varphi & \varphi^{2} & \varphi^{3}\end{array}\right], \mathbf{B}=\left[\begin{array}{llll}0 & 1 & 0 & 0 \\ 0 & 0 & 2 & 0 \\ 0 & 0 & 0 & 3 \\ 0 & 0 & 0 & 0\end{array}\right], A_{1=}\left[\begin{array}{l}a_{10} \\ a_{11} \\ a_{12} \\ a_{13}\end{array}\right], A_{2=}\left[\begin{array}{l}a_{20} \\ a_{21} \\ a_{22} \\ a_{23}\end{array}\right], A_{3=}\left[\begin{array}{l}a_{30} \\ a_{31} \\ a_{32} \\ a_{33}\end{array}\right]$

Thus, with $\rho(\varphi) \tau(\varphi)=\omega(\varphi)$, the augmented matrix $[\tilde{\mathbf{W}} ; \tilde{\mathbf{G}}]$ is calculated and the approximate solutions are found as

$$
\begin{aligned}
& \lambda \cong \lambda_{3}(\varphi)=\lambda_{0}+\mu_{0} \varphi-\frac{3 \mathrm{~L}_{1}}{2 K} \varphi^{2}+\frac{9 L_{2}}{8 \pi K} \varphi^{3}, \\
& \mu \cong \mu_{3}(\varphi)=\mu_{0}+\left(-\lambda_{0}+\delta_{0} \omega(0)\right) \varphi-\frac{9 \mathrm{~L}_{3}}{8 \pi K} \varphi^{2}+\frac{9 L_{4}}{8 \pi^{2} K} \varphi^{3}, \\
& \delta \cong \delta_{3}(\varphi)=\delta_{0}-\mu_{0} \omega(0) \varphi+\frac{3 \mathrm{~L}_{5}}{8 \pi K} \varphi^{2}-\frac{9 L_{6}}{8 \pi^{2} K} \varphi^{3},
\end{aligned}
$$

where

$$
\begin{aligned}
K= & 2187+\left(324+48 \pi^{2}\right) \pi^{2}+\left(432+32 \pi^{2}\right) \pi^{2}(\omega(2 \pi / 3))^{2}-\left(216-32 \pi^{2}\right) \pi^{2} \omega(2 \pi / 3) \omega(4 \pi / 3) \\
& +\left(108+32 \pi^{2}\right) \pi^{2}(\omega(4 \pi / 3))^{2}+48 \pi^{4}(\omega(2 \pi / 3))^{2}(\omega(4 \pi / 3))^{2}, \\
\mathrm{~L}_{1}= & \left(729+216 \pi^{2}\right) \lambda_{0}+72 \mu_{0} \pi^{3}-\left(486-90 \pi^{2}\right) \delta_{0} \omega(0)-\left(486+144 \pi^{2}\right) \delta_{0} \omega(2 \pi / 3) \\
& +\left(135+40 \pi^{2}\right) \mu_{0} \pi \omega(0) \omega(2 \pi / 3)+\left(216+16 \pi^{2}\right) \mu_{0} \pi(\omega(2 \pi / 3))^{2}-36 \delta_{0} \pi^{2} \omega(0)(\omega(2 \pi / 3))^{2} \\
& +\left(243+18 \pi^{2}\right) \delta_{0} \omega(4 \pi / 3)-\left(54+4 \pi^{2}\right) \mu_{0} \pi \omega(0) \omega(4 \pi / 3)-18 \delta_{0} \pi^{2} \omega(0) \omega(2 \pi / 3) \omega(4 \pi / 3) \\
& -\left(\left(243-48 \pi^{2}\right) \mu_{0}-108 \lambda_{0} \pi\right) \pi \omega(2 \pi / 3) \omega(4 \pi / 3)-36 \delta_{0} \pi^{2}(\omega(2 \pi / 3))^{2} \omega(4 \pi / 3) \\
& +12 \mu_{0} \pi^{3} \omega(0)(\omega(2 \pi / 3))^{2} \omega(4 \pi / 3)-\left(\left(54-44 \pi^{2}\right) \mu_{0} \pi-108 \lambda_{0} \pi^{2}\right)(\omega(4 \pi / 3))^{2} \\
& -36 \delta_{0} \pi^{2} \omega(0)(\omega(4 \pi / 3))^{2}-90 \delta_{0} \pi^{2} \omega(2 \pi / 3)(\omega(4 \pi / 3))^{2}+24 \mu_{0} \pi^{3} \omega(0) \omega(2 \pi / 3)(\omega(4 \pi / 3))^{2} \\
& +36 \mu_{0} \pi^{3}(\omega(2 \pi / 3))^{2}(\omega(4 \pi / 3))^{2},
\end{aligned}
$$




$$
\begin{aligned}
& L_{2}=-\left(324-48 \mu_{0} \pi^{2}\right) \mu_{0} \pi+216 \lambda_{0} \pi^{2}-\left(243-108 \pi^{2}\right) \delta_{0} \omega(0)-144 \delta_{0} \pi^{2} \omega(2 \pi / 3) \\
& +40 \mu_{0} \pi^{3} \omega(0) \omega(2 \pi / 3)-48 \delta_{0} \pi^{2} \omega(0)(\omega(2 \pi / 3))^{2}+\left(243+36 \pi^{2}\right) \delta_{0} \omega(2 \pi / 3) \\
& -\left(54+8 \pi^{2}\right) \mu_{0} \pi \omega(0) \omega(2 \pi / 3)-\left(\left(216-40 \pi^{2}\right) \mu_{0} \pi-144 \lambda_{0} \pi^{2}\right) \omega(2 \pi / 3) \omega(4 \pi / 3) \\
& -36 \delta_{0} \pi^{2} \omega(0) \omega(2 \pi / 3) \omega(4 \pi / 3)-48 \delta_{0} \pi^{2}(\omega(2 \pi / 3))^{2} \omega(4 \pi / 3)+ \\
& +16 \mu_{0} \pi^{3} \omega(0)(\omega(2 \pi / 3))^{2} \omega(4 \pi / 3)-\left(\left(54-24 \pi^{2}\right) \mu_{0} \pi-72 \lambda_{0} \pi^{2}\right)(\omega(4 \pi / 3))^{2} \\
& -24 \delta_{0} \pi^{2} \omega(0)(\omega(4 \pi / 3))^{2}-60 \delta_{0} \pi^{2} \omega(2 \pi / 3)(\omega(4 \pi / 3))^{2} \\
& +16 \mu_{0} \pi^{3} \omega(0) \omega(2 \pi / 3)(\omega(4 \pi / 3))^{2}+16 \mu_{0} \pi^{3}(\omega(2 \pi / 3))^{2}(\omega(4 \pi / 3))^{2}, \\
& L_{3}=-96 \lambda_{0} \pi^{4}+\left(972+288 \pi^{2}\right) \mu_{0} \pi+\left(2187+432 \pi^{2}+96 \pi^{4}\right) \delta_{0} \omega(0)-\left(2916+432 \delta_{0} \pi^{2}\right) \delta_{0} \omega(2 \pi / 3) \\
& +\left(810+120 \pi^{2}\right) \mu_{0} \pi \omega(0) \omega(2 \pi / 3)+\left(\left(1296+96 \pi^{2}\right) \mu_{0}-\left(864+64 \lambda_{0} \pi^{2}\right) \lambda_{0} \pi\right) \pi(\omega(2 \pi / 3))^{2} \\
& +\left(792+64 \delta_{0} \pi^{2}\right) \delta_{0} \pi^{2} \omega(0)(\omega(2 \pi / 3))^{2}-\left(432-64 \pi^{2}\right) \delta_{0} \pi^{2} \omega(0) \omega(2 \pi / 3) \omega(4 \pi / 3) \\
& -\left(\left(810-168 \pi^{2}\right) \mu_{0} \pi-\left(648-64 \pi^{2}\right) \lambda_{0} \pi^{2}\right) \omega(2 \pi / 3) \omega(4 \pi / 3)+729 \delta_{0} \omega(4 \pi / 3) \\
& -162 \mu_{0} \pi \omega(0) \omega(4 \pi / 3)-72 \delta_{0} \pi^{2}(\omega(2 \pi / 3))^{2} \omega(4 \pi / 3)+24 \mu_{0} \pi^{3} \omega(0)(\omega(2 \pi / 3))^{2} \omega(4 \pi / 3) \\
& -\left(\left(162-192 \pi^{2}\right) \mu_{0} \pi-\left(216-64 \pi^{2}\right) \lambda_{0} \pi^{2}\right)(\omega(4 \pi / 3))^{2}+\left(72+64 \pi^{2}\right) \delta_{0} \pi^{2} \omega(0)(\omega(4 \pi / 3))^{2} \\
& -360 \delta_{0} \pi^{2} \omega(2 \pi / 3)(\omega(4 \pi / 3))^{2}+96 \mu_{0} \pi^{3} \omega(0) \omega(2 \pi / 3)(\omega(4 \pi / 3))^{2} \\
& +\left(168 \mu_{0}-96 \lambda_{0} \pi\right) \pi^{3}(\omega(2 \pi / 3))^{2}(\omega(4 \pi / 3))^{2}+96 \delta_{0} \pi^{4} \omega(0)(\omega(2 \pi / 3))^{2}(\omega(4 \pi / 3))^{2}, \\
& L_{4}=216 \mu_{0} \pi^{3}+\left(324-48 \pi^{2}\right) \lambda_{0} \pi^{2}+\left(729+54 \pi^{2}+48 \pi^{4}\right) \delta_{0} \omega(0)-\left(1458+432 \delta_{0} \pi^{2}\right) \delta_{0} \omega(2 \pi / 3) \\
& +\left(405+120 \pi^{2}\right) \mu_{0} \pi \omega(0) \omega(2 \pi / 3)+\left(\left(648+48 \pi^{2}\right) \mu_{0} \pi-\left(432+32 \pi^{2}\right) \lambda_{0} \pi^{2}\right)(\omega(2 \pi / 3))^{2} \\
& +\left(324+32 \pi^{2}\right) \delta_{0} \pi^{2} \omega(0)(\omega(2 \pi / 3))^{2}+72 \mu_{0} \pi^{3} \omega(0) \omega(2 \pi / 3)(\omega(4 \pi / 3))^{2} \\
& -\left(162+12 \pi^{2}\right) \mu_{0} \pi \omega(0) \omega(4 \pi / 3)-\left(\left(729-144 \pi^{2}\right) \mu_{0} \pi-\left(540-32 \pi^{2}\right) \lambda_{0} \pi^{2}\right) \omega(2 \pi / 3) \omega(4 \pi / 3) \\
& -\left(270-32 \pi^{2}\right) \delta_{0} \pi^{2} \omega(0) \omega(2 \pi / 3) \omega(4 \pi / 3)-108 \delta_{0} \pi^{2}(\omega(2 \pi / 3))^{2} \omega(4 \pi / 3) \\
& +36 \mu_{0} \pi^{3} \omega(0)(\omega(2 \pi / 3))^{2} \omega(4 \pi / 3)-\left(\left(162-132 \pi^{2}\right) \mu_{0} \pi-\left(216-32 \pi^{2}\right) \lambda_{0} \pi^{2}\right)(\omega(4 \pi / 3))^{2} \\
& +32 \delta_{0} \pi^{4} \omega(0)(\omega(4 \pi / 3))^{2}-270 \delta_{0} \pi^{2} \omega(2 \pi / 3)(\omega(4 \pi / 3))^{2}+\left(729+54 \pi^{2}\right) \delta_{0} \omega(4 \pi / 3) \\
& +\left(108 \mu_{0} \pi^{3}-48 \lambda_{0} \pi^{4}\right)(\omega(2 \pi / 3))^{2}(\omega(4 \pi / 3))^{2}+48 \delta_{0} \pi^{4} \omega(0)(\omega(2 \pi / 3))^{2}(\omega(4 \pi / 3))^{2} \text {, }
\end{aligned}
$$




$$
\begin{aligned}
& L_{5}=\left(6561+972 \pi^{2}+144 \pi^{4}\right) \mu_{0} \omega(0)-\left(\left(8748-648 \pi^{2}-96 \pi^{4}\right) \mu_{0}-\left(5832+432 \pi^{2}\right) \lambda_{0} \pi\right) \omega(2 \pi / 3) \\
& -\left(2430+72 \pi^{2}\right) \delta_{0} \pi \omega(0) \omega(2 \pi / 3)-\left(3888+288 \pi^{2}\right) \delta_{0} \pi(\omega(2 \pi / 3))^{2} \\
& +\left(2376+176 \pi^{2}\right) \mu_{0} \pi^{2} \omega(0)(\omega(2 \pi / 3))^{2}+\left(486-288 \pi^{2}\right) \delta_{0} \pi \omega(0) \omega(4 \pi / 3) \\
& +\left(\left(2187-1620 \pi^{2}+48 \pi^{4}\right) \mu_{0}-\left(2916-432 \pi^{2}\right) \lambda_{0} \pi\right) \omega(4 \pi / 3) \\
& +\left(2430-360 \pi^{2}\right) \delta_{0} \pi \omega(2 \pi / 3) \omega(4 \pi / 3)-\left(1296-192 \pi^{2}\right) \mu_{0} \pi^{2} \omega(0) \omega(2 \pi / 3) \omega(4 \pi / 3) \\
& -\left(216+16 \pi^{2}\right) \mu_{0} \pi^{2}(\omega(2 \pi / 3))^{2} \omega(4 \pi / 3)-72 \delta_{0} \pi^{3} \omega(0)(\omega(2 \pi / 3))^{2} \omega(4 \pi / 3) \\
& +\left(486+144 \pi^{2}\right) \delta_{0} \pi(\omega(4 \pi / 3))^{2}+\left(216+64 \pi^{2}\right) \mu_{0} \pi^{2} \omega(0)(\omega(4 \pi / 3))^{2} \\
& \text { - }\left(\left(1080-160 \pi^{2}\right) \mu_{0}-864 \lambda_{0} \pi\right) \pi^{2} \omega(2 \pi / 3)(\omega(4 \pi / 3))^{2}-288 \delta_{0} \pi^{3} \omega(0) \omega(2 \pi / 3)(\omega(4 \pi / 3))^{2} \\
& -504 \delta_{0} \pi^{3}(\omega(2 \pi / 3))^{2}(\omega(4 \pi / 3))^{2}+288 \mu_{0} \pi^{4} \omega(0)(\omega(2 \pi / 3))^{2}(\omega(4 \pi / 3))^{2} \text {, } \\
& L_{6}=\left(729+108 \pi^{2}+16 \pi^{4}\right) \mu_{0} \omega(0)-\left(\left(1458-108 \pi^{2}-16 \pi^{4}\right) \mu_{0}-\left(972+72 \pi^{2}\right) \lambda_{0} \pi\right) \omega(2 \pi / 3) \\
& \text { - }\left(405+12 \pi^{2}\right) \delta_{0} \pi \omega(0) \omega(2 \pi / 3)-\left(648+48 \delta_{0} \pi^{2}\right) \delta_{0} \pi(\omega(2 \pi / 3))^{2} \\
& +\left(324+24 \pi^{2}\right) \mu_{0} \pi^{2} \omega(0)(\omega(2 \pi / 3))^{2}+\left(\left(729-540 \pi^{2}+16 \pi^{4}\right) \mu_{0}-\left(972-144 \pi^{2}\right) \lambda \pi\right) \omega(4 \pi / 3) \\
& +\left(162-96 \pi^{2}\right) \delta_{0} \pi \omega(0) \omega(4 \pi / 3)+\left(729-108 \pi^{2}\right) \delta_{0} \pi \omega(2 \pi / 3) \omega(4 \pi / 3) \\
& -\left(270-40 \pi^{2}\right) \mu_{0} \pi^{2} \omega(0) \omega(2 \pi / 3) \omega(4 \pi / 3)-\left(108+8 \pi^{2}\right) \mu_{0} \pi^{2}(\omega(2 \pi / 3))^{2} \omega(4 \pi / 3) \\
& -36 \delta_{0} \pi^{3} \omega(0)(\omega(2 \pi / 3))^{2} \omega(4 \pi / 3)+\left(162+48 \pi^{2}\right) \delta_{0} \pi(\omega(4 \pi / 3))^{2} \\
& \text { - }\left(\left(270-40 \pi^{2}\right) \mu_{0}-216 \lambda_{0} \pi\right) \pi^{2} \omega(2 \pi / 3)(\omega(4 \pi / 3))^{2}-72 \delta_{0} \pi^{3} \omega(0) \omega(2 \pi / 3)(\omega(4 \pi / 3))^{2} \\
& -108 \delta_{0} \pi^{3}(\omega(2 \pi / 3))^{2}(\omega(4 \pi / 3))^{2}+48 \mu_{0} \pi^{4} \omega(0)(\omega(2 \pi / 3))^{2}(\omega(4 \pi / 3))^{2} \text {. }
\end{aligned}
$$

Similarly, in order to obtain the approximate solutions with the Hermite matrix collocation method, the equations (7.1), (7.2) and (7.3) are written in the matrix form as follows:

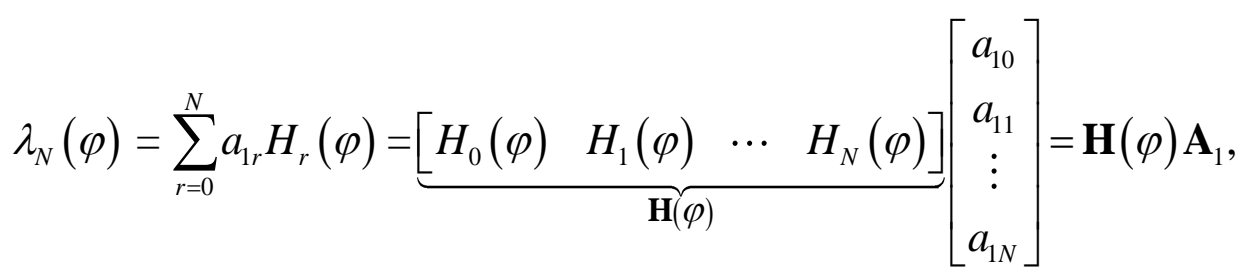

$\mathbf{A}_{1}$ 


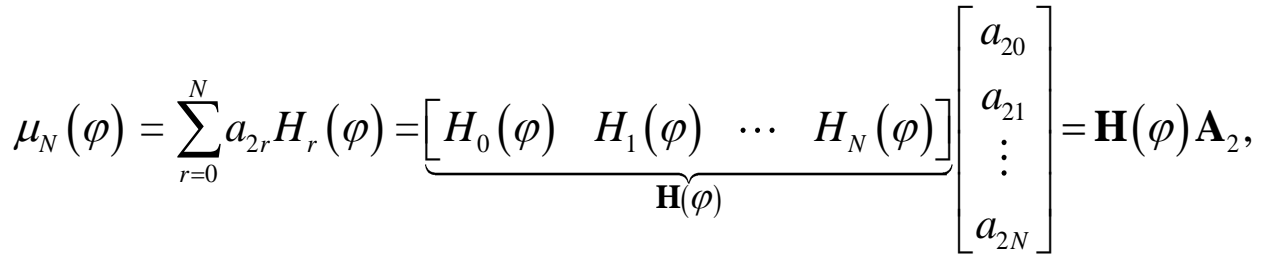

$$
\begin{aligned}
& \mathbf{A}_{2}
\end{aligned}
$$

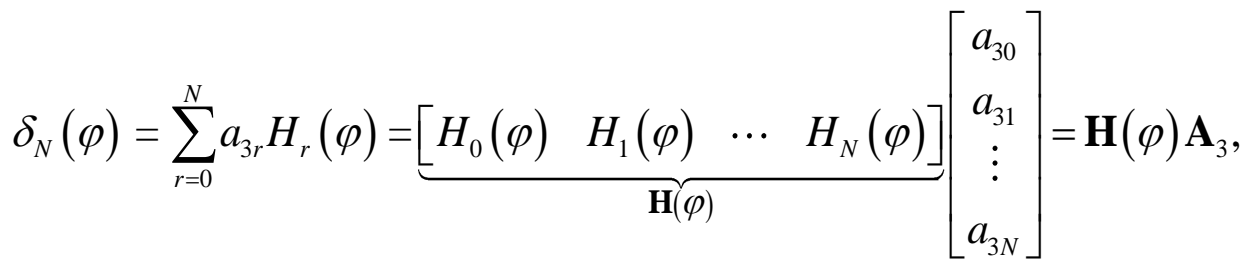

$$
\begin{aligned}
& \mathbf{A}_{3}
\end{aligned}
$$

where the matrix $\mathbf{H}(\varphi)$ becomes

$$
\mathbf{H}(\varphi)=\mathbf{X}(\varphi) \mathbf{F}
$$

with the help of (11), for

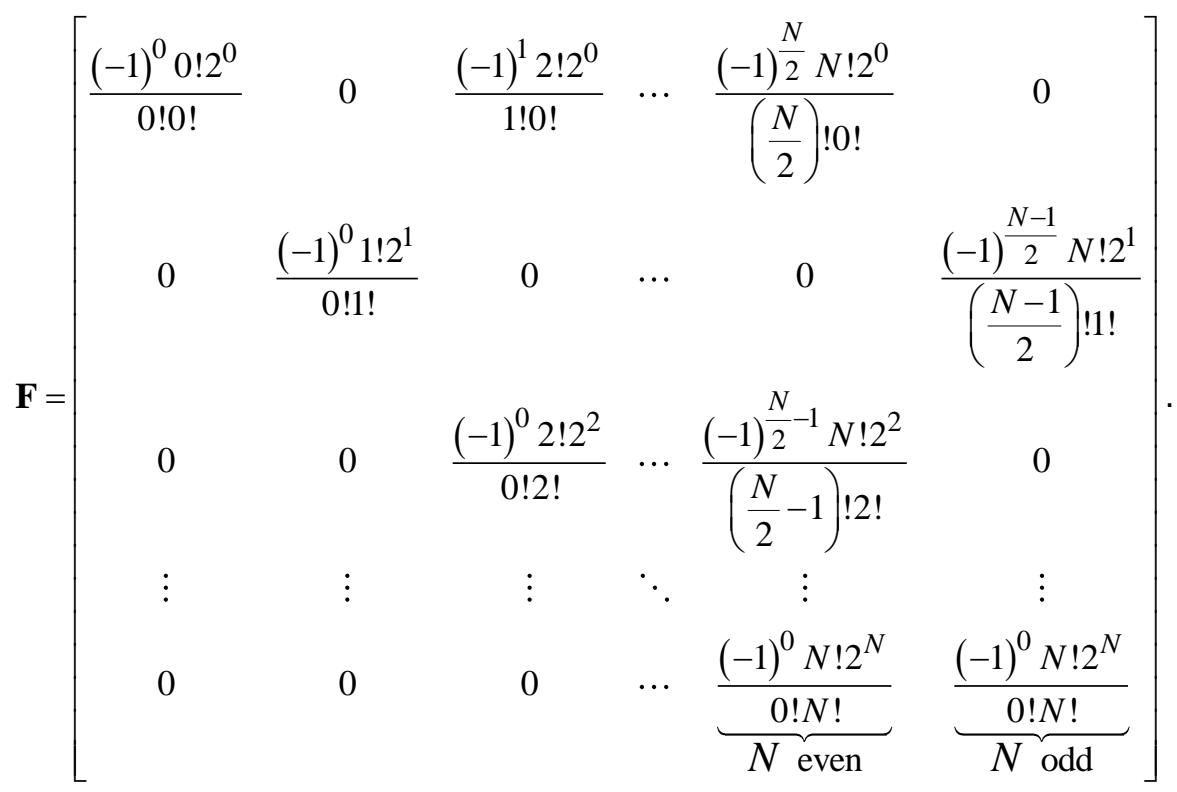

The equation (23) is written in the equations (20), (21) and (22) to obtain the following equalities:

$$
\begin{aligned}
\lambda_{N}(\varphi) & =\mathbf{X}(\varphi) \mathbf{F} \mathbf{A}_{1}, \\
\mu_{N}(\varphi) & =\mathbf{X}(\varphi) \mathbf{F} \mathbf{A}_{2}, \\
\delta_{N}(\varphi) & =\mathbf{X}(\varphi) \mathbf{F} \mathbf{A}_{3} .
\end{aligned}
$$

By arranging the expression (24), the matrix relation 


$$
\underbrace{\left[\begin{array}{c}
\lambda_{N}(\varphi) \\
\mu_{N}(\varphi) \\
\delta_{N}(\varphi)
\end{array}\right]}_{\boldsymbol{\Lambda}(\varphi)}=\underbrace{\left[\begin{array}{ccc}
\mathbf{X}(\varphi) & 0 & 0 \\
0 & \mathbf{X}(\varphi) & 0 \\
0 & 0 & \mathbf{X}(\varphi)
\end{array}\right]}_{\overline{\mathbf{X}}(\varphi)} \underbrace{\left[\begin{array}{ccc}
\mathbf{F} & 0 & 0 \\
0 & \mathbf{F} & 0 \\
0 & 0 & \mathbf{F}
\end{array}\right]}_{\overline{\mathbf{F}}}\left[\begin{array}{l}
\mathbf{A}_{1} \\
\mathbf{A}_{2} \\
\mathbf{A}_{3}
\end{array}\right] \Rightarrow \boldsymbol{\Lambda}(\varphi)=\overline{\mathbf{X}}(\varphi) \overline{\mathbf{F}} \mathbf{A}
$$

is obtained. This relation (25) is written as

$$
\begin{aligned}
\lambda_{N}^{\prime}(\varphi) & =\mathbf{X}^{\prime}(\varphi) \mathbf{F} \mathbf{A}_{1}=\mathbf{X}(\varphi) \mathbf{B F A}_{1}, \\
\mu_{N}^{\prime}(\varphi) & =\mathbf{X}^{\prime}(\varphi) \mathbf{F} \mathbf{A}_{2}=\mathbf{X}(\varphi) \mathbf{B F A} \mathbf{A}_{2}, \\
\delta_{N}^{\prime}(\varphi) & =\mathbf{X}^{\prime}(\varphi) \mathbf{F A}_{3}=\mathbf{X}(\varphi) \mathbf{B F A}_{3}
\end{aligned}
$$

using the equation (12). The expression (26) is arranged in matrix form and expressed as follows:

$$
\begin{aligned}
& \underbrace{\left[\begin{array}{c}
\lambda_{N}^{\prime}(\varphi) \\
\mu_{N}^{\prime}(\varphi) \\
\delta_{N}^{\prime}(\varphi)
\end{array}\right]}_{\boldsymbol{\Lambda}^{\prime}(\varphi)}=\underbrace{\left[\begin{array}{ccc}
\mathbf{X}(\varphi) & 0 & 0 \\
0 & \mathbf{X}(\varphi) & 0 \\
0 & 0 & \mathbf{X}(\varphi)
\end{array}\right]}_{\overline{\mathbf{X}}(\varphi)} \underbrace{\left[\begin{array}{ccc}
\mathbf{B} & 0 & 0 \\
0 & \mathbf{B} & 0 \\
0 & 0 & \mathbf{B}
\end{array}\right]}_{\overline{\mathbf{B}}} \underbrace{\left[\begin{array}{ccc}
\mathbf{F} & 0 & 0 \\
0 & \mathbf{F} & 0 \\
0 & 0 & \mathbf{F}
\end{array}\right]}_{\overline{\mathbf{F}}} \underbrace{\left[\begin{array}{c}
\mathbf{A}_{1} \\
\mathbf{A}_{2} \\
\mathbf{A}_{3}
\end{array}\right]}_{\mathbf{A}} \\
& \Rightarrow \boldsymbol{\Lambda}^{\prime}(\varphi)=\overline{\mathbf{X}}(\varphi) \overline{\mathbf{B}} \overline{\mathbf{F}} \mathbf{A} .
\end{aligned}
$$

By writing the equations (25) and (27) into the equation (9), the matrix relation

$$
\mathbf{P}_{1}(\varphi) \overline{\mathbf{X}}(\varphi) \overline{\mathbf{B}} \overline{\mathbf{F}} \mathbf{A}+\mathbf{P}_{0}(\varphi) \overline{\mathbf{X}}(\varphi) \overline{\mathbf{F}} \mathbf{A}=\mathbf{G}(\varphi)
$$

is found. The collocation points (16) are written in the matrix relation (28) to obtain the fundamental matrix relation

$$
\left\{\mathbf{P}_{1} \overline{\mathbf{X}} \overline{\mathbf{B}}^{*} \overline{\mathbf{F}}+\mathbf{P}_{0} \overline{\mathbf{X}}^{*} \overline{\mathbf{I}}^{*} \overline{\mathbf{F}}\right\} \mathbf{A}=\mathbf{G}
$$

and this expression can be written briefly in form

$$
\mathbf{W}_{H} \mathbf{A}=\mathbf{G} \text { or }\left[\mathbf{W}_{H} ; \mathbf{G}\right] ; \quad \mathbf{W}_{H}=\mathbf{P}_{1} \overline{\mathbf{X}} \overline{\mathbf{B}}^{*} \overline{\mathbf{F}}+\mathbf{P}_{0} \overline{\mathbf{X}} \overline{\mathbf{I}}^{*} \overline{\mathbf{F}} .
$$

On the other hand, using equation (24) in the initial conditions (5), the following expression is obtained:

$$
\begin{aligned}
& \lambda(0)=\lambda_{0} \Rightarrow \mathbf{X}(0) \mathbf{F} \mathbf{A}_{1}=\lambda_{0}, \\
& \mu(0)=\mu_{0} \Rightarrow \mathbf{X}(0) \mathbf{F} \mathbf{A}_{2}=\mu_{0}, \\
& \delta(0)=\delta_{0} \Rightarrow \mathbf{X}(0) \mathbf{F} \mathbf{A}_{3}=\delta_{0} .
\end{aligned}
$$

The expression (30) is arranged and the matrix relations of the conditions 


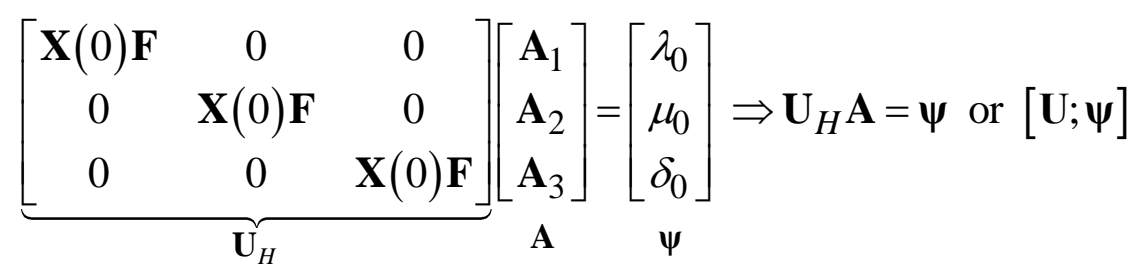

are obtained. ). In order to obtain the solutions of the differential equation system (4) under initial conditions (5), the augmented matrix $\left[\tilde{\mathbf{W}}_{H} ; \tilde{\mathbf{G}}\right]$ is obtained by deleting any three rows of the augmented matrix $\left[\mathbf{W}_{H} ; \mathbf{G}\right]$ and writing the augmented matrix $\left[\mathbf{U}_{H} ; \boldsymbol{\psi}\right]$ of the conditions instead. If $\operatorname{rank}\left(\tilde{\mathbf{W}}_{H}\right)=\operatorname{rank}\left(\tilde{\mathbf{W}}_{H} ; \tilde{\mathbf{G}}\right)=3(N+1)$, the solution A of augmented matrix $\left[\tilde{\mathbf{W}}_{H} ; \tilde{\mathbf{G}}\right]$ is obtained. Thus, the unknown coefficients of the Hermite polynomial are obtained and the approximate solutions are found in the form of the truncated Hermite series (7).

If specially, the interval $0 \leq \varphi \leq 2 \pi$ and truncation limit $N=3$ are selected; the collocation points are $\varphi_{0}=0, \varphi_{1}=\frac{2 \pi}{3}, \varphi_{2}=\frac{4 \pi}{3}, \varphi_{3}=2 \pi$ and the matrices are

$\mathbf{X}(\varphi)=\left[\begin{array}{llll}1 & \varphi & \varphi^{2} & \varphi^{3}\end{array}\right], \quad \mathbf{F}=\left[\begin{array}{cccc}1 & 0 & -2 & 0 \\ 0 & 2 & 0 & -12 \\ 0 & 0 & 4 & 0 \\ 0 & 0 & 0 & 8\end{array}\right], \mathbf{B}=\left[\begin{array}{cccc}0 & 1 & 0 & 0 \\ 0 & 0 & 2 & 0 \\ 0 & 0 & 0 & 3 \\ 0 & 0 & 0 & 0\end{array}\right]$

$A_{1}=\left[\begin{array}{l}a_{10} \\ a_{11} \\ a_{12} \\ a_{13}\end{array}\right], \quad A_{2}=\left[\begin{array}{l}a_{20} \\ a_{21} \\ a_{22} \\ a_{23}\end{array}\right], \quad A_{3}=\left[\begin{array}{l}a_{30} \\ a_{31} \\ a_{32} \\ a_{33}\end{array}\right]$.

Thus, with $\rho(\varphi) \tau(\varphi)=\omega(\varphi)$, the augmented matrix $\left[\tilde{\mathbf{W}}_{H} ; \tilde{\mathbf{G}}\right]$ is calculated and the approximate solutions are found as

$$
\begin{aligned}
& \lambda \cong \tilde{\lambda}_{3}(\varphi)=\frac{N_{1}}{4 M}+\frac{N_{2}}{32 \pi M} 2 \varphi-\frac{3 N_{3}}{8 M}\left(4 \varphi^{2}-2\right)+\frac{9 N_{4}}{64 \pi M}\left(8 \varphi^{3}-12 \varphi\right), \\
& \mu \cong \tilde{\mu}_{3}(\varphi)=\frac{N_{5}}{16 \pi M}+\frac{N_{6}}{32 \pi^{2} M} 2 \varphi+\frac{9 N_{7}}{32 \pi M}\left(4 \varphi^{2}-2\right)-\frac{9 N_{8}}{64 \pi^{2} M}\left(8 \varphi^{3}-12 \varphi\right), \\
& \delta \cong \tilde{\delta}_{3}(\varphi)=\frac{N_{9}}{16 \pi M}+\frac{N_{10}}{32 \pi^{2} M} 2 \varphi+\frac{3 N_{11}}{32 \pi M}\left(4 \varphi^{2}-2\right)-\frac{9 N_{12}}{64 \pi^{2} M}\left(8 \varphi^{3}-12 \varphi\right),
\end{aligned}
$$

where

$$
\begin{aligned}
M= & 2187+\left(324+48 \pi^{2}\right) \pi^{2}+\left(432+32 \pi^{2}\right) \pi^{2}(\omega(2 \pi / 3))^{2}-\left(216-32 \pi^{2}\right) \pi^{2} \omega(2 \pi / 3) \omega(4 \pi / 3) \\
& +\left(108+32 \pi^{2}\right) \pi^{2}(\omega(4 \pi / 3))^{2}+48 \pi^{4}(\omega(2 \pi / 3))^{2}(\omega(4 \pi / 3))^{2},
\end{aligned}
$$




$$
\begin{aligned}
& N_{1}=-216 \mu_{0} \pi^{3}+\left(6561+648 \pi^{2}+192 \pi^{4}\right) \lambda_{0}+\left(1458+270 \pi^{2}\right) \delta_{0} \omega(0)+\left(1458+432 \pi^{2}\right) \delta_{0} \omega(2 \pi / 3) \\
& -\left(405+120 \pi^{2}\right) \mu_{0} \pi \omega(0) \omega(2 \pi / 3)-\left(\left(648+48 \pi^{2}\right) \mu_{0} \pi-\left(1728+128 \pi^{2}\right) \lambda_{0} \pi^{2}\right)(\omega(2 \pi / 3))^{2} \\
& +108 \delta_{0} \pi^{2} \omega(0)(\omega(2 \pi / 3))^{2}-\left(729+54 \pi^{2}\right) \delta_{0} \omega(4 \pi / 3)+\left(162+12 \pi^{2}\right) \mu_{0} \pi \omega(0) \omega(4 \pi / 3) \\
& +\left(\left(729-144 \pi^{2}\right) \mu_{0} \pi-\left(1188-128 \pi^{2}\right) \lambda_{0} \pi^{2}\right) \omega(2 \pi / 3) \omega(4 \pi / 3)+54 \delta_{0} \pi^{2} \omega(0) \omega(2 \pi / 3) \omega(4 \pi / 3) \\
& +108 \delta_{0} \pi^{2}(\omega(2 \pi / 3))^{2} \omega(4 \pi / 3)-36 \mu_{0} \pi^{3} \omega(0)(\omega(2 \pi / 3))^{2} \omega(4 \pi / 3) \\
& +\left(\left(162-132 \pi^{2}\right) \mu_{0} \pi+\left(108+128 \pi^{2}\right) \lambda_{0} \pi^{2}\right)(\omega(4 \pi / 3))^{2}+108 \delta_{0} \pi^{2} \omega(0)(\omega(4 \pi / 3))^{2} \\
& +270 \delta_{0} \pi^{2} \omega(2 \pi / 3)(\omega(4 \pi / 3))^{2}-72 \mu_{0} \pi^{3} \omega(0) \omega(2 \pi / 3)(\omega(4 \pi / 3))^{2} \\
& -\left(108 \mu_{0}-192 \lambda_{0} \pi\right) \pi^{3}(\omega(2 \pi / 3))^{2}(\omega(4 \pi / 3))^{2} \text {, } \\
& N_{2}=5832 \lambda_{0} \pi^{2}+\left(26244+6480 \pi^{2}+768 \pi^{4}\right) \mu_{0} \pi-\left(6561+2916 \delta_{0} \pi^{2}\right) \delta_{0} \omega(0)-3888 \delta_{0} \pi^{2} \omega(2 \pi / 3) \\
& +1080 \mu_{0} \pi^{3} \omega(0) \omega(2 \pi / 3)+\left(6912+512 \pi^{2}\right) \mu_{0} \pi^{3}(\omega(2 \pi / 3))^{2}-1296 \delta_{0} \pi^{2} \omega(0)(\omega(2 \pi / 3))^{2} \\
& +\left(6561+972 \delta_{0} \pi^{2}\right) \delta_{0} \omega(4 \pi / 3)-\left(1458-216 \pi^{2}\right) \mu_{0} \pi \omega(0) \omega(4 \pi / 3) \\
& -\left(\left(5832+2376 \pi^{2}-512 \mu_{0} \pi^{4}\right) \mu_{0}-3888 \lambda_{0} \pi\right) \pi \omega(2 \pi / 3) \omega(4 \pi / 3) \\
& -972 \delta_{0} \pi^{2} \omega(0) \omega(2 \pi / 3) \omega(4 \pi / 3)-1296 \delta_{0} \pi^{2}(\omega(2 \pi / 3))^{2} \omega(4 \pi / 3) \\
& +432 \mu_{0} \pi^{3} \omega(0)(\omega(2 \pi / 3))^{2} \omega(4 \pi / 3)-\left(\left(1458-2376 \pi^{2}-512 \pi^{4}\right) \mu_{0}-1944 \lambda_{0} \pi\right) \pi(\omega(4 \pi / 3))^{2} \\
& \text { - } 648 \delta_{0} \pi^{2} \omega(0)(\omega(4 \pi / 3))^{2}-1620 \delta_{0} \pi^{2} \omega(2 \pi / 3)(\omega(4 \pi / 3))^{2} \\
& +432 \mu_{0} \pi^{3} \omega(0) \omega(2 \pi / 3)(\omega(4 \pi / 3))^{2}+\left(432+768 \pi^{2}\right) \mu_{0} \pi^{3}(\omega(2 \pi / 3))^{2}(\omega(4 \pi / 3))^{2} \text {, } \\
& N_{3}=72 \mu_{0} \pi^{3}+\left(729+216 \pi^{2}\right) \lambda_{0}-\left(486+90 \pi^{2}\right) \delta_{0} \omega(0)-\left(486+144 \pi^{2}\right) \delta_{0} \omega(2 \pi / 3) \\
& +\left(135+40 \pi^{2}\right) \mu_{0} \pi \omega(0) \omega(2 \pi / 3)+\left(216+16 \pi^{2}\right) \mu_{0} \pi(\omega(2 \pi / 3))^{2}-36 \delta_{0} \pi^{2} \omega(0)(\omega(2 \pi / 3))^{2} \\
& +\left(243+18 \pi^{2}\right) \delta_{0} \omega(4 \pi / 3)-\left(54+4 \pi^{2}\right) \mu_{0} \pi \omega(0) \omega(4 \pi / 3)-18 \delta_{0} \pi^{2} \omega(0) \omega(2 \pi / 3) \omega(4 \pi / 3) \\
& -\left(\left(243-48 \pi^{2}\right) \mu_{0}-108 \lambda_{0} \pi\right) \pi \omega(2 \pi / 3) \omega(4 \pi / 3)-36 \delta_{0} \pi^{2}(\omega(2 \pi / 3))^{2} \omega(4 \pi / 3) \\
& +12 \mu_{0} \pi^{3} \omega(0)(\omega(2 \pi / 3))^{2} \omega(4 \pi / 3)-\left(\left(54-44 \pi^{2}\right) \mu_{0}-108 \lambda_{0} \pi\right) \pi(\omega(4 \pi / 3))^{2} \\
& -36 \delta_{0} \pi^{2} \omega(0)(\omega(4 \pi / 3))^{2}-90 \delta_{0} \pi^{2} \omega(2 \pi / 3)(\omega(4 \pi / 3))^{2}+24 \mu_{0} \pi^{3} \omega(0) \omega(2 \pi / 3)(\omega(4 \pi / 3))^{2}
\end{aligned}
$$

$+36 \mu_{0} \pi^{3}(\omega(2 \pi / 3))^{2}(\omega(4 \pi / 3))^{2}$ 


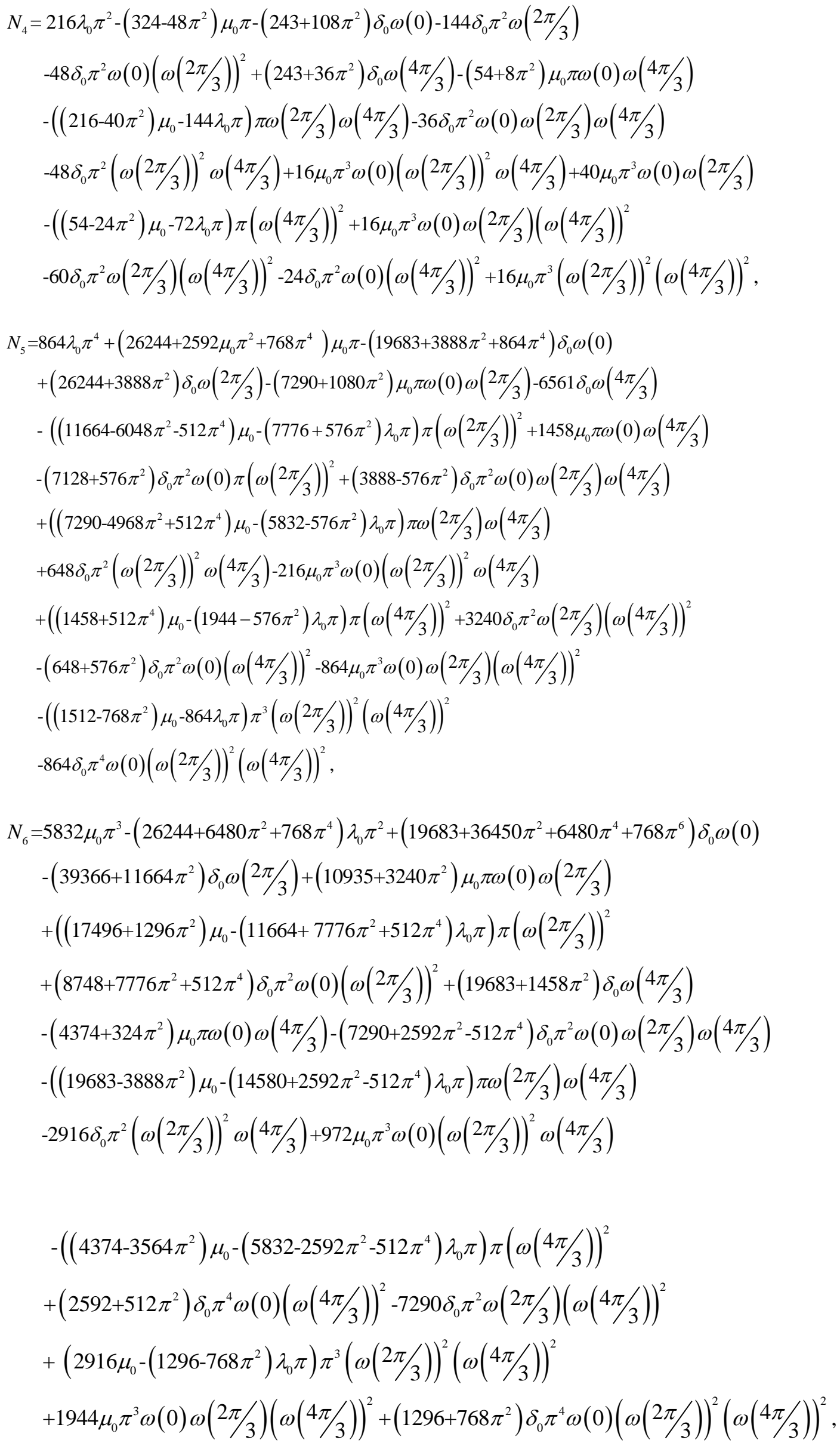




$$
\begin{aligned}
& N_{7}=96 \lambda_{0} \pi^{4}-\left(972+288 \pi^{2}\right) \mu_{0} \pi-\left(2187+432 \pi^{2}+96 \pi^{4}\right) \delta_{0} \omega(0)+\left(2916+432 \pi^{2}\right) \delta_{0} \omega(2 \pi / 3) \\
& -\left(810+120 \pi^{2}\right) \mu_{0} \pi \omega(0) \omega(2 \pi / 3)-\left(1296 \mu_{0} \pi-864 \lambda_{0} \pi^{2}+96 \mu_{0} \pi^{3}-64 \lambda_{0} \pi^{4}\right)(\omega(2 \pi / 3))^{2} \\
& -\left(792+64 \pi^{2}\right) \delta_{0} \pi^{2} \omega(0)(\omega(2 \pi / 3))^{2}-729 \delta_{0} \omega(4 \pi / 3)+162 \mu_{0} \pi \omega(0) \omega(4 \pi / 3) \\
& +\left(\left(810-168 \pi^{2}\right) \mu_{0}-\left(648-64 \pi^{2}\right) \lambda_{0} \pi\right) \pi \omega(2 \pi / 3) \omega(4 \pi / 3)+72 \delta_{0} \pi^{2}(\omega(2 \pi / 3))^{2} \omega(4 \pi / 3) \\
& +\left(432-64 \pi^{2}\right) \delta_{0} \pi^{2} \omega(0) \omega(2 \pi / 3) \omega(4 \pi / 3)-24 \mu_{0} \pi^{3} \omega(0)(\omega(2 \pi / 3))^{2} \omega(4 \pi / 3) \\
& +\left(162 \mu_{0} \pi-216 \lambda_{0} \pi^{2}-192 \mu_{0} \pi^{3}+64 \lambda_{0} \pi^{4}\right)(\omega(4 \pi / 3))^{2}-\left(72+64 \pi^{2}\right) \delta_{0} \pi^{2} \omega(0)(\omega(4 \pi / 3))^{2} \\
& +360 \delta_{0} \pi^{2} \omega(2 \pi / 3)(\omega(4 \pi / 3))^{2}-96 \mu_{0} \pi^{3} \omega(0) \omega(2 \pi / 3)(\omega(4 \pi / 3))^{2} \\
& -\left(168 \mu_{0}-96 \lambda_{0} \pi\right) \pi^{3}(\omega(2 \pi / 3))^{2}(\omega(4 \pi / 3))^{2}-96 \delta_{0} \pi^{4} \omega(0)(\omega(2 \pi / 3))^{2}(\omega(4 \pi / 3))^{2} \text {, } \\
& N_{8}=-216 \mu_{0} \pi^{3}-\left(324-48 \pi^{2}\right) \lambda_{0} \pi^{2}-\left(729+54 \pi^{2}+48 \pi^{4}\right) \delta_{0} \omega(0)+\left(1458+432 \pi^{2}\right) \delta_{0} \omega(2 \pi / 3) \\
& -\left(405+120 \pi^{2}\right) \mu_{0} \pi \omega(0) \omega(2 \pi / 3)-\left(\left(648+48 \pi^{2}\right) \mu_{0}-\left(432+32 \pi^{2}\right) \lambda_{0} \pi\right) \pi(\omega(2 \pi / 3))^{2} \\
& -\left(324+32 \pi^{2}\right) \delta_{0} \pi^{2} \omega(0)(\omega(2 \pi / 3))^{2}-\left(729+54 \pi^{2}\right) \delta_{0} \omega(4 \pi / 3) \\
& +\left(162+12 \pi^{2}\right) \mu_{0} \pi \omega(0) \omega(4 \pi / 3)+\left(\left(729-144 \pi^{2}\right) \mu_{0}-\left(540-32 \pi^{2}\right) \lambda_{0} \pi\right) \pi \omega(2 \pi / 3) \omega(4 \pi / 3) \\
& +\left(270-32 \pi^{2}\right) \delta_{0} \pi^{2} \omega(0) \omega(2 \pi / 3) \omega(4 \pi / 3)+108 \delta_{0} \pi^{2}(\omega(2 \pi / 3))^{2} \omega(4 \pi / 3) \\
& -36 \mu_{0} \pi^{3} \omega(0)(\omega(2 \pi / 3))^{2} \omega(4 \pi / 3)+\left(\left(162-132 \pi^{2}\right) \mu_{0}-\left(216-32 \pi^{2}\right) \lambda_{0} \pi\right) \pi(\omega(4 \pi / 3))^{2} \\
& -32 \delta_{0} \pi^{4} \omega(0)(\omega(4 \pi / 3))^{2}+270 \delta_{0} \pi^{2} \omega(2 \pi / 3)(\omega(4 \pi / 3))^{2}-72 \mu_{0} \pi^{3} \omega(0) \omega(2 \pi / 3)(\omega(4 \pi / 3))^{2} \\
& -\left(108 \mu_{0}-48 \lambda_{0} \pi\right) \pi^{3}(\omega(2 \pi / 3))^{2}(\omega(4 \pi / 3))^{2}-48 \delta_{0} \pi^{4} \omega(0)(\omega(2 \pi / 3))^{2}(\omega(4 \pi / 3))^{2} \text {, } \\
& N_{9}=\left(34992+5184 \pi^{2}+768 \pi^{4}\right) \delta_{0} \pi+\left(19683+2916 \pi^{2}+432 \pi^{4}\right) \mu_{0} \omega(0) \\
& -\left(\left(26244-1944 \pi^{2}-288 \pi^{4}\right) \mu_{0}-\left(17496+1296 \pi^{2}\right) \lambda_{0} \pi\right) \omega(2 \pi / 3) \\
& -\left(7290 \delta_{0} \pi+216 \delta_{0} \pi^{3}\right) \omega(0) \omega(2 \pi / 3)-\left(11664-6048 \pi^{2}-512 \pi^{4}\right) \delta_{0} \pi(\omega(2 \pi / 3))^{2} \\
& +\left(7128+528 \pi^{2}\right) \mu_{0} \pi^{2} \omega(0)(\omega(2 \pi / 3))^{2}+\left(1458-864 \pi^{2}\right) \delta_{0} \pi \omega(0) \omega(4 \pi / 3)
\end{aligned}
$$




$$
\begin{aligned}
& +\left(\left(6561-4860 \pi^{2}+144 \pi^{4}\right) \mu_{0}-\left(8748-1296 \pi^{2}\right) \lambda_{0} \pi\right) \omega(4 \pi / 3) \\
& +\left(7290-4536 \pi^{2}+512 \pi^{4}\right) \delta_{0} \pi \omega(2 \pi / 3) \omega(4 \pi / 3)-\left(648+48 \pi^{2}\right) \mu_{0} \pi^{2}(\omega(2 \pi / 3))^{2} \omega(4 \pi / 3) \\
& -\left(3888-576 \pi^{2}\right) \mu_{0} \pi^{2} \omega(0) \omega(2 \pi / 3) \omega(4 \pi / 3)-216 \delta_{0} \pi^{3} \omega(0)(\omega(2 \pi / 3))^{2} \omega(4 \pi / 3) \\
& +\left(1458+2160 \pi^{2}+512 \pi^{4}\right) \delta_{0} \pi(\omega(4 \pi / 3))^{2}+\left(648+192 \pi^{2}\right) \mu_{0} \pi^{2} \omega(0)(\omega(4 \pi / 3))^{2} \\
& -\left(\left(3240-480 \pi^{2}\right) \mu_{0}-2592 \lambda_{0} \pi\right) \pi^{2} \omega(2 \pi / 3)(\omega(4 \pi / 3))^{2}-864 \delta_{0} \pi^{3} \omega(0) \omega(2 \pi / 3)(\omega(4 \pi / 3))^{2} \\
& -\left(1512-768 \pi^{2}\right) \delta_{0} \pi^{3}(\omega(2 \pi / 3))^{2}(\omega(4 \pi / 3))^{2}+864 \mu_{0} \pi^{4} \omega(0)(\omega(2 \pi / 3))^{2}(\omega(4 \pi / 3))^{2} \text {, } \\
& N_{10}=-\left(19683+37908 \pi^{2}+5616 \pi^{4}+768 \pi^{6}\right) \mu_{0} \omega(0)+\left(10935+324 \pi^{2}\right) \delta_{0} \pi \omega(0) \omega(2 \pi / 3) \\
& +\left(\left(39366-2916 \pi^{2}-432 \pi^{4}\right) \mu_{0}-\left(26244+1944 \pi^{2}\right) \lambda_{0} \pi\right) \omega(2 \pi / 3) \\
& +\left(17496+1296 \pi^{2}\right) \delta_{0} \pi(\omega(2 \pi / 3))^{2}-\left(8748+7560 \pi^{2}+512 \pi^{4}\right) \mu_{0} \pi^{2} \omega(0)(\omega(2 \pi / 3))^{2} \\
& -\left(\left(19683-14580 \pi^{2}+432 \pi^{4}\right) \mu_{0}-\left(26244-3888 \pi^{2}\right) \lambda_{0} \pi\right) \omega(4 \pi / 3) \\
& -\left(4374 \pi-2592 \pi^{2}\right) \delta_{0} \pi \omega(0) \omega(4 \pi / 3)-\left(19683-2916 \pi^{2}\right) \delta_{0} \pi \omega(2 \pi / 3) \omega(4 \pi / 3) \\
& +\left(7290+2376 \pi^{2}-512 \pi^{4}\right) \mu_{0} \pi^{2} \omega(0) \omega(2 \pi / 3) \omega(4 \pi / 3)+972 \delta_{0} \pi^{3} \omega(0)(\omega(2 \pi / 3))^{2} \omega(4 \pi / 3) \\
& +\left(2916+216 \pi^{2}\right) \mu_{0} \pi^{2}(\omega(2 \pi / 3))^{2} \omega(4 \pi / 3)-\left(4374+1296 \pi^{2}\right) \delta_{0} \pi(\omega(4 \pi / 3))^{2} \\
& -\left(1728+512 \pi^{2}\right) \mu_{0} \pi^{4} \omega(0)(\omega(4 \pi / 3))^{2}+1944 \delta_{0} \pi^{3} \omega(0) \omega(2 \pi / 3)(\omega(4 \pi / 3))^{2} \\
& +\left(\left(7290-1080 \pi^{2}\right) \mu_{0}-5832 \lambda_{0} \pi\right) \pi^{2} \omega(2 \pi / 3)(\omega(4 \pi / 3))^{2}+2916 \delta_{0} \pi^{3}(\omega(2 \pi / 3))^{2}(\omega(4 \pi / 3))^{2} \\
& -\left(1296+768 \pi^{2}\right) \mu_{0} \pi^{4} \omega(0)(\omega(2 \pi / 3))^{2}(\omega(4 \pi / 3))^{2} \text {, } \\
& N_{11}=\left(6561 \mu_{0}+972 \mu_{0} \pi^{2}+144 \mu_{0} \pi^{4}\right) \omega(0)-\left(2430+72 \pi^{2}\right) \delta_{0} \pi \omega(0) \omega(2 \pi / 3) \\
& -\left(\left(8748-648 \pi^{2}-96 \pi^{4}\right) \mu_{0}-\left(5832+432 \pi^{2}\right) \lambda_{0} \pi\right) \omega(2 \pi / 3)-\left(3888+288 \pi^{2}\right) \delta_{0} \pi(\omega(2 \pi / 3))^{2} \\
& +\left(2376+176 \pi^{2}\right) \mu_{0} \pi^{2} \omega(0)(\omega(2 \pi / 3))^{2}+\left(486-288 \pi^{2}\right) \delta_{0} \pi \omega(0) \omega(4 \pi / 3) \\
& +\left(\left(2187-1620 \pi^{2}+48 \pi^{4}\right) \mu_{0}-\left(2916-432 \pi^{2}\right) \lambda_{0} \pi\right) \omega(4 \pi / 3) \\
& +\left(2430-360 \pi^{2}\right) \delta_{0} \pi \omega(2 \pi / 3) \omega(4 \pi / 3)-\left(1296-192 \pi^{2}\right) \mu_{0} \pi^{2} \omega(0) \omega(2 \pi / 3) \omega(4 \pi / 3) \\
& -\left(216+16 \pi^{2}\right) \mu_{0} \pi^{2}(\omega(2 \pi / 3))^{2} \omega(4 \pi / 3)-72 \delta_{0} \pi^{3} \omega(0)(\omega(2 \pi / 3))^{2} \omega(4 \pi / 3) \\
& +\left(486 \delta_{0} \pi+144 \pi^{2}\right) \delta_{0} \pi(\omega(4 \pi / 3))^{2}+\left(216+64 \pi^{2}\right) \mu_{0} \pi^{2} \omega(0)(\omega(4 \pi / 3))^{2} \\
& -\left(\left(1080-160 \pi^{2}\right) \mu_{0}-864 \lambda_{0} \pi\right) \pi^{2} \omega(2 \pi / 3)(\omega(4 \pi / 3))^{2}-288 \delta_{0} \pi^{3} \omega(0) \omega(2 \pi / 3)(\omega(4 \pi / 3))^{2} \\
& -504 \delta_{0} \pi^{3}(\omega(2 \pi / 3))^{2}(\omega(4 \pi / 3))^{2}+288 \mu_{0} \pi^{4} \omega(0)(\omega(2 \pi / 3))^{2}(\omega(4 \pi / 3))^{2} \text {, }
\end{aligned}
$$




$$
\begin{aligned}
N_{12}= & \left(729+108 \pi^{2}+16 \pi^{4}\right) \mu_{0} \omega(0)-\left(\left(1458-108 \pi^{2}-16 \pi^{4}\right) \mu_{0}-\left(972+72 \pi^{2}\right) \lambda_{0} \pi\right) \omega(2 \pi / 3) \\
& -\left(405+12 \pi^{2}\right) \delta_{0} \pi \omega(0) \omega(2 \pi / 3)-\left(648+48 \pi^{2}\right) \delta_{0} \pi(\omega(2 \pi / 3))^{2} \\
& +\left(324+24 \pi^{2}\right) \mu_{0} \pi^{2} \omega(0)(\omega(2 \pi / 3))^{2}+\left(\left(729-540 \pi^{2}+16 \pi^{4}\right) \mu_{0}-\left(972-144 \pi^{2}\right) \lambda_{0} \pi\right) \omega(4 \pi / 3) \\
& +\left(162-96 \pi^{2}\right) \delta_{0} \pi \omega(0) \omega(4 \pi / 3)+\left(729-108 \pi^{2}\right) \delta_{0} \pi \omega(2 \pi / 3) \omega(4 \pi / 3) \\
& -\left(270-40 \pi^{2}\right) \mu_{0} \pi^{2} \omega(0) \omega(2 \pi / 3) \omega(4 \pi / 3)-\left(108+8 \pi^{2}\right) \mu_{0} \pi^{2}(\omega(2 \pi / 3))^{2} \omega(4 \pi / 3) \\
& -36 \delta_{0} \pi^{3} \omega(0)(\omega(2 \pi / 3))^{2} \omega(4 \pi / 3)+\left(162+48 \pi^{2}\right) \delta_{0} \pi(\omega(4 \pi / 3))^{2} \\
& -\left(\left(270-40 \pi^{2}\right) \mu_{0}-216 \lambda_{0} \pi\right) \pi^{2} \omega(2 \pi / 3)(\omega(4 \pi / 3))^{2}-72 \delta_{0} \pi^{3} \omega(0) \omega(2 \pi / 3)(\omega(4 \pi / 3))^{2} \\
& -108 \delta_{0} \pi^{3}(\omega(2 \pi / 3))^{2}(\omega(4 \pi / 3))^{2}+48 \mu_{0} \pi^{4} \omega(0)(\omega(2 \pi / 3))^{2}(\omega(4 \pi / 3))^{2} .
\end{aligned}
$$

\subsection{Numerical example}

In this section, the coefficient functions determining the unit speed curve of constant width, with $\rho \tau=s+1$ in the interval $0 \leq s \leq 1$ for the initial conditions $\lambda_{0}=0, \mu_{0}=1$ and $\delta_{0}=1$ in Euclidean space are obtained by the Taylor and Hermite matrix collocation methods, respectively. Then, the error analysis based on the residual function is performed for the approximate solutions obtained. These approximate solutions obtained are compared in Table 1,2,3.

The approximate solutions obtained for $N=3, N=5$ and $N=7$ using the Taylor matrix collocation method are as follows:

for $N=3$

$$
\begin{aligned}
& \lambda(s) \cong \lambda_{3}(s)=0.9999999999999999 s+0.5985738509053473 s^{2}-0.47408102706958144 s^{3} \\
& \begin{array}{l}
\mu(s) \cong \mu_{3}(s)=0.9999999999999999+s-0.5350784230606188 s^{2}-0.8871646581481254 s^{3} \\
\delta(s) \cong \delta_{3}(s)=1-s-1.2566008599285403 s^{2}+0.5491128202022643 s^{3}
\end{array} \\
& \text { for } N=5
\end{aligned}
$$

$$
\begin{aligned}
\lambda(s) \cong \lambda_{5}(s)= & 0.9999999999999998 s+0.49250588557529645 s^{2}-0.11952315355595314 s^{3} \\
& -0.3181290799304512 s^{4}+0.04919037394703688 s^{5} \\
\mu(s) \cong \mu_{5}(s)= & 0.9999999999999998+s-0.5146968445158518 s^{2}-0.7260704762538329 s^{3} \\
& -0.5346850495047698 s^{4}+0.39031845961997397 s^{5} \\
\delta(s) \cong \delta_{5}(s)= & 1-s-0.9877836096763788 s^{2}-0.24160768612561084 s^{3} \\
+ & 0.49385459950474087 s^{4}+0.10693214210324661 s^{5}
\end{aligned}
$$

for $N=7$ 


$$
\begin{aligned}
\lambda(s) \cong \lambda_{\neg}(s)= & 0.9999999999999996 s+0.5000865521703856 s^{2}-0.16739595712666583 s^{3} \\
& -0.2057192060821741 s^{4}-0.05408968495019506 s^{5} \\
& +0.013512030901242048 s^{6}+0.018734462020299388 s^{7}
\end{aligned}
$$

$$
\begin{aligned}
\mu(s) \cong \mu_{7}(s) & =0.9999999999999996+1.0000000000000002 s-0.4992191319357816 s^{2} \\
& -0.842008700746969 s^{3}-0.2098366610982572 s^{4}-0.01994742068043524 s^{5} \\
& +0.2159977032559501 s^{6}-0.028285489704628533 s^{7}
\end{aligned}
$$$$
\delta(s) \cong \delta_{7}(s)=1.0000000000000002-0.9999999999999994 s-0.9996852666151173 s^{2}
$$$$
-0.1705831091688063 s^{3}+0.35344165958397417 s^{4}+0.16246166375485893 s^{5}
$$$$
+0.10962296497529832 s^{6}-0.08651958885372914 s^{7} \text {, }
$$

The approximate solutions obtained for $N=3, N=5$ and $N=7$ using the Hermite matrix collocation method are as follows:

for $N=3$

$$
\begin{aligned}
\lambda(s) \cong \tilde{\lambda}_{3}(s)= & 0.29008101454017987+0.3072011291784602 s \\
& +0.14504050727008994\left(4 s^{2}-2\right)-0.05773323923512832\left(8 s^{3}-12 s\right) \\
\mu(s) \cong \tilde{\mu}_{3}(s)= & 0.7347319509403789-0.28259246528575555 s \\
& -0.1326340245298106\left(4 s^{2}-2\right)-0.10688270544047962\left(8 s^{3}-12 s\right) \\
\delta(s) \cong \tilde{\delta}_{3}(s)= & 0.3949690913203008-0.19456428743728055 s \\
& -0.30251545433984955\left(4 s^{2}-2\right)+0.06711964271355995\left(8 s^{3}-12 s\right),
\end{aligned}
$$

for $N=5$

$$
\begin{aligned}
\lambda(s) \cong \tilde{\lambda}_{5}(s)= & 0.012318861249204727+1.0022713888544788 s \\
& -0.11174551888657161\left(4 s^{2}-2\right)+0.01651857121935095\left(8 s^{3}-12 s\right) \\
& -0.019650824918528997\left(16 s^{4}-48 s^{2}+12\right) \\
& +0.0016329288814811045\left(32 s^{5}-160 s^{3}+120 s\right) \\
\mu(s) \cong \tilde{\mu}_{5}(s)= & 0.36754687296520827+1.3051167777785104 s \\
& -0.5056732529180102\left(4 s^{2}-2\right)+0.14518574076882657\left(8 s^{3}-12 s\right) \\
& -0.03157444823343574\left(16 s^{4}-48 s^{2}+12\right) \\
& +0.011975934262061737\left(32 s^{5}-160 s^{3}+120 s\right)
\end{aligned}
$$




$$
\begin{aligned}
\delta(s) \cong \tilde{\delta}_{5}(s) & =0.8758834679939984-0.9880154669007108 s \\
& +0.12430998528544601\left(4 s^{2}-2\right)+0.030614144321288794\left(8 s^{3}-12 s\right) \\
& +0.031061375214741137\left(16 s^{4}-48 s^{2}+12\right) \\
& +0.0029615433229681384\left(32 s^{5}-160 s^{3}+120 s\right),
\end{aligned}
$$

for $N=7$

$$
\begin{aligned}
\lambda(s) \cong \tilde{\lambda}_{7}(s) & =0.12495968298682486+0.7746601272200926 s \\
& +0.014586777497836281\left(4 s^{2}-2\right)+0.0027528269425966694\left(8 s^{3}-12 s\right) \\
& -0.005543254165133217\left(16 s^{4}-48 s^{2}+12\right) \\
& +0.004094757595023809\left(32 s^{5}-160 s^{3}+120 s\right) \\
& +0.00024389231674628074\left(64 s^{6}-480 s^{4}+720 s^{2}-120\right) \\
& +0.00013868875911415997\left(128 s^{7}-1344 s^{5}+3360 s^{3}-1680 s\right) \\
\mu(s) \cong \tilde{\mu}_{7}(s) & =0.9876009314660955-0.6904583698791326 s \\
& +0.3138916819180195\left(4 s^{2}-2\right)-0.21000266617543992\left(8 s^{3}-12 s\right) \\
& +0.08690602290970782\left(16 s^{4}-48 s^{2}+12\right) \\
& -0.01021269434254584\left(32 s^{5}-160 s^{3}+120 s\right) \\
& +0.003355748687887913\left(64 s^{6}-480 s^{4}+720 s^{2}-120\right) \\
& -0.00023568434338056856\left(128 s^{7}-1344 s^{5}+3360 s^{3}-1680 s\right) \\
\delta(s) \cong \tilde{\delta}_{7}(s) & =0.9505763881943383-1.7101442942316458 s \\
& +0.29592097030628567\left(4 s^{2}-2\right)-0.18959770156292177\left(8 s^{3}-12 s\right) \\
& +0.06933290995376652\left(16 s^{4}-48 s^{2}+12\right) \\
& -0.022271825448578256\left(32 s^{5}-160 s^{3}+120 s\right) \\
& +0.0015894113918913705\left(64 s^{6}-480 s^{4}+720 s^{2}-120\right) \\
& -0.0006592803150630799\left(128 s^{7}-1344 s^{5}+3360 s^{3}-1680 s\right) . \\
&
\end{aligned}
$$

When these approximate solutions and their derivatives are put into place in the system of differential equations that characterize curves of constant width, the equation is approximately provided. For this reason, it is written as

$$
\begin{aligned}
& R 1_{N}\left(s_{i}\right)=\left|\lambda^{\prime}\left(s_{i}\right)-\mu\left(s_{i}\right)\right| \cong 0, \\
& R 2_{N}\left(s_{i}\right)=\left|\mu^{\prime}\left(s_{i}\right)-\rho\left(s_{i}\right) \tau\left(s_{i}\right) \delta\left(s_{i}\right)+\lambda\left(s_{i}\right)\right| \cong 0, \\
& R 3_{N}\left(s_{i}\right)=\left|\delta^{\prime}\left(s_{i}\right)+\rho\left(s_{i}\right) \tau\left(s_{i}\right) \mu\left(s_{i}\right)\right| \cong 0
\end{aligned}
$$


in the interval $0 \leq s_{i} \leq \chi$, with $i=0,1, \ldots, N$, where $R 1_{N}\left(s_{i}\right) \leq 10^{-k 1_{i}}, R 2_{N}\left(s_{i}\right) \leq 10^{-k 2_{i}}$ and $R 3_{N}\left(s_{i}\right) \leq 10^{-k 3_{i}}$ $\left(k 1_{i}, k 2_{i}, k 3_{i} \in Z^{+}\right.$) should be provided. $R 1_{N}(s), R 2_{N}(s)$ and $R 3_{N}(s)$ comparisons are made in Table 4,5,6 for the obtained approximate solutions.

Table 1 Comparison of the obtained approximate solutions.

\begin{tabular}{|c|c|c|c|c|c|c|}
\hline \multirow[b]{2}{*}{$\mathbf{s}$} & \multicolumn{3}{|c|}{ Taylor Matrix Collocation Method } & \multicolumn{3}{|c|}{ Hermite Matrix Collocation Method } \\
\hline & $\lambda_{3}(s)$ & $\lambda_{5}(s)$ & $\lambda_{7}(s)$ & $\tilde{\lambda}_{3}(s)$ & $\tilde{\lambda}_{5}(s)$ & $\tilde{\lambda}_{7}(s)$ \\
\hline 0.1 & 0.10551 & 0.10477 & 0.10481 & 0.10534 & 0.10480 & 0.10481 \\
\hline 0.3 & 0.34107 & 0.33864 & 0.33870 & 0.33974 & 0.33875 & 0.33870 \\
\hline 0.5 & 0.59038 & 0.58984 & 0.58990 & 0.58730 & 0.58990 & 0.58990 \\
\hline 0.7 & 0.83069 & 0.83221 & 0.83227 & 0.82585 & 0.83217 & 0.83227 \\
\hline 0.9 & 1.03924 & 1.03212 & 1.03227 & 1.03323 & 1.03241 & 1.03227 \\
\hline 1.0 & 1.12449 & 1.10404 & 1.10513 & 1.11830 & 1.10498 & 1.10512 \\
\hline
\end{tabular}

Table 2 Comparison of the obtained approximate solutions.

\begin{tabular}{|c|c|c|c|c|c|c|}
\hline \multirow{2}{*}{$\mathbf{S}$} & \multicolumn{3}{|c|}{ Taylor Matrix Collocation Method } & \multicolumn{3}{|c|}{ Hermite Matrix Collocation Method } \\
\hline & $\mu_{3}(s)$ & $\mu_{5}(s)$ & $\mu_{7}(s)$ & $\tilde{\mu}_{3}(s)$ & $\tilde{\mu}_{5}(s)$ & $\tilde{\mu}_{7}(s)$ \\
\hline 0.1 & 1.09376 & 1.09408 & 1.09414 & 1.09384 & 1.09413 & 1.09414 \\
\hline 0.3 & 1.22789 & 1.23069 & 1.23074 & 1.22917 & 1.23082 & 1.23074 \\
\hline 0.5 & 1.25533 & 1.25935 & 1.25936 & 1.26048 & 1.25929 & 1.25936 \\
\hline 0.7 & 1.13351 & 1.13598 & 1.13592 & 1.14675 & 1.13577 & 1.13592 \\
\hline 0.9 & 0.81984 & 0.83346 & 0.83361 & 0.84692 & 0.83392 & 0.83361 \\
\hline 1.0 & 0.57775 & 0.61486 & 0.61670 & 0.61440 & 0.61625 & 0.61661 \\
\hline
\end{tabular}

Table 3 Comparison of the obtained approximate solutions.

\begin{tabular}{|c|c|c|c|c|c|c|}
\hline \multirow[b]{2}{*}{$\mathbf{s}$} & \multicolumn{3}{|c|}{ Taylor Matrix Collocation Method } & \multicolumn{3}{|c|}{ Hermite Matrix Collocation Method } \\
\hline & $\delta_{3}(s)$ & $\delta_{5}(s)$ & $\delta_{7}(s)$ & $\tilde{\delta}_{3}(s)$ & $\tilde{\delta}_{5}(s)$ & $\tilde{\delta}_{7}(s)$ \\
\hline 0.1 & 0.88798 & 0.88993 & 0.88987 & 0.88843 & 0.88988 & 0.88986 \\
\hline 0.3 & 0.60173 & 0.60883 & 0.60874 & 0.60559 & 0.60864 & 0.60874 \\
\hline 0.5 & 0.25448 & 0.25706 & 0.25696 & 0.26460 & 0.25698 & 0.25696 \\
\hline 0.7 & -0.12738 & -0.13033 & -0.13041 & -0.10875 & -0.13018 & -0.13041 \\
\hline 0.9 & -0.51754 & -0.48907 & -0.48939 & -0.48870 & -0.48976 & -0.48939 \\
\hline 1.0 & -0.70748 & -0.62860 & -0.63126 & -0.67310 & -0.63088 & -0.63127 \\
\hline
\end{tabular}

Table $4 R 1_{N}(s)$ comparison for the obtained approximate solutions.

\begin{tabular}{|c|c|c|c|c|c|c|}
\hline \multirow[b]{2}{*}{$\mathbf{s}$} & \multicolumn{3}{|c|}{ Taylor Matrix Collocation Method } & \multicolumn{3}{|c|}{ Hermite Matrix Collocation Method } \\
\hline & $R 1_{3}(s)$ & $R 1_{5}(s)$ & $R 1_{7}(s)$ & $R 1_{3}(s)$ & $R 1_{5}(s)$ & $R 1_{7}(s)$ \\
\hline 0.1 & $1.17 \mathrm{E}-02$ & $4.09 \mathrm{E}-04$ & $1.62 \mathrm{E}-06$ & $8.33 \mathrm{E}-03$ & 1.89E-05 & $1.20 \mathrm{E}-06$ \\
\hline 0.3 & $3.25 \mathrm{E}-03$ & $1.75 \mathrm{E}-04$ & $1.53 \mathrm{E}-07$ & $5.77 \mathrm{E}-03$ & $2.29 \mathrm{E}-04$ & $2.32 \mathrm{E}-06$ \\
\hline
\end{tabular}




\begin{tabular}{|l|l|l|l|l|r|r|r|}
\hline 0.5 & $1.23 \mathrm{E}-02$ & $1.75 \mathrm{E}-04$ & $4.22 \mathrm{E}-07$ & & $2.67 \mathrm{E}-02$ & $7.48 \mathrm{E}-04$ & $3.68 \mathrm{E}-06$ \\
\hline 0.7 & $7.59 \mathrm{E}-03$ & $4.09 \mathrm{E}-04$ & $3.58 \mathrm{E}-07$ & & $1.34 \mathrm{E}-02$ & $5.34 \mathrm{E}-04$ & $5.43 \mathrm{E}-06$ \\
\hline 0.9 & $1.05 \mathrm{E}-01$ & $3.68 \mathrm{E}-03$ & $1.45 \mathrm{E}-05$ & & $7.50 \mathrm{E}-02$ & $1.70 \mathrm{E}-04$ & $1.08 \mathrm{E}-05$ \\
\hline 1.0 & $1.97 \mathrm{E}-01$ & $1.49 \mathrm{E}-02$ & $1.73 \mathrm{E}-04$ & & $1.60 \mathrm{E}-01$ & $7.48 \mathrm{E}-03$ & $5.15 \mathrm{E}-05$ \\
\hline
\end{tabular}

Table $5 R 2_{N}(s)$ comparison for the obtained approximate solutions.

\begin{tabular}{|l|l|l|l|l|l|l|}
\hline \multirow{4}{*}{$\mathbf{s}$} & \multicolumn{3}{|l|}{ Taylor Matrix Collocation Method } & \multicolumn{3}{l|}{ Hermite Matrix Collocation Method } \\
\cline { 2 - 7 } & $R 2_{3}(s)$ & $R 2_{5}(s)$ & $R 2_{7}(s)$ & $R 2_{3}(s)$ & $R 2_{5}(s)$ & $R 2_{7}(s)$ \\
\hline 0.1 & $4.90 \mathrm{E}-03$ & $8.14 \mathrm{E}-04$ & $1.51 \mathrm{E}-05$ & $3.69 \mathrm{E}-03$ & $3.64 \mathrm{E}-05$ & $1.04 \mathrm{E}-05$ \\
\hline 0.3 & $1.76 \mathrm{E}-03$ & $3.58 \mathrm{E}-04$ & $1.52 \mathrm{E}-06$ & $3.28 \mathrm{E}-03$ & $4.53 \mathrm{E}-04$ & $2.15 \mathrm{E}-05$ \\
\hline 0.5 & $8.19 \mathrm{E}-03$ & $3.68 \mathrm{E}-04$ & $4.45 \mathrm{E}-06$ & $1.85 \mathrm{E}-02$ & $1.51 \mathrm{E}-03$ & $3.61 \mathrm{E}-05$ \\
\hline 0.7 & $5.98 \mathrm{E}-03$ & $8.82 \mathrm{E}-04$ & $3.99 \mathrm{E}-06$ & $1.10 \mathrm{E}-02$ & $1.11 \mathrm{E}-03$ & $5.63 \mathrm{E}-05$ \\
\hline 0.9 & $9.63 \mathrm{E}-02$ & $8.14 \mathrm{E}-03$ & $1.71 \mathrm{E}-04$ & $7.09 \mathrm{E}-02$ & $3.61 \mathrm{E}-04$ & $1.18 \mathrm{E}-04$ \\
\hline 1.0 & $1.92 \mathrm{E}-01$ & $3.34 \mathrm{E}-02$ & $2.09 \mathrm{E}-03$ & $1.61 \mathrm{E}-01$ & $1.60 \mathrm{E}-02$ & $5.77 \mathrm{E}-04$ \\
\hline
\end{tabular}

Table $6 R 3_{N}(s)$ comparison for the obtained approximate solutions.

\begin{tabular}{|c|c|c|c|c|c|c|}
\hline \multirow[b]{2}{*}{$\mathbf{s}$} & \multicolumn{3}{|c|}{ Taylor Matrix Collocation Method } & \multicolumn{3}{|c|}{ Hermite Matrix Collocation Method } \\
\hline & $R 3_{3}(s)$ & $R 3_{5}(s)$ & $R 3_{7}(s)$ & $R 3_{3}(s)$ & $R 3_{5}(s)$ & $R 3_{7}(s)$ \\
\hline 0.1 & $3.17 \mathrm{E}-02$ & 7.09E-04 & 5.73E-06 & $2.26 \mathrm{E}-02$ & $3.37 \mathrm{E}-05$ & $3.62 \mathrm{E}-06$ \\
\hline 0.3 & $9.44 \mathrm{E}-03$ & $3.39 \mathrm{E}-04$ & $5.11 \mathrm{E}-07$ & $1.68 \mathrm{E}-02$ & $4.54 \mathrm{E}-04$ & $6.56 \mathrm{E}-06$ \\
\hline 0.5 & $3.82 \mathrm{E}-02$ & $3.74 \mathrm{E}-04$ & $1.32 \mathrm{E}-06$ & 8.33E-02 & $1.63 \mathrm{E}-03$ & $9.64 \mathrm{E}-06$ \\
\hline 0.7 & $2.50 \mathrm{E}-02$ & $9.54 \mathrm{E}-04$ & $1.05 \mathrm{E}-06$ & $4.47 \mathrm{E}-02$ & $1.27 \mathrm{E}-03$ & $1.31 \mathrm{E}-05$ \\
\hline 0.9 & $3.69 \mathrm{E}-01$ & 9.33E-03 & $3.99 \mathrm{E}-05$ & $2.64 \mathrm{E}-01$ & $4.39 \mathrm{E}-04$ & $2.39 \mathrm{E}-05$ \\
\hline 1.0 & 7.10E-01 & $3.94 \mathrm{E}-02$ & $4.56 \mathrm{E}-04$ & $5.80 \mathrm{E}-01$ & $2.00 \mathrm{E}-02$ & $1.09 \mathrm{E}-04$ \\
\hline
\end{tabular}

\section{Result and Discussion}

According to the error analysis maked, it is observed that the amount of error decreases as the $\mathrm{N}$ value, which is the truncation limit for both solutions, increases. In addition, it is obtained that the approximate solution obtained by Hermite matrix collocation method approaches with less error than the Taylor matrix collocation method. Therefore, it can be stated that Hermite matrix collocation method is a more suitable method for the system characterizing this curve type. Expressing the curve of constant width with differential equations and these approximate solutions will allow this curve type to be preferred more in practice.

\section{Conclusion}

In this article, firstly, curves of constant width are characterized with a system of differential equations. Then, two different approximate solutions of this system are calculated using Hermite and Taylor matrix collocation methods. In the future studies, characterizations of this curve type in different spaces such as Dual space and Galilean space can be obtained. The approximate solution can be obtained with similar methods for characterizations in different spaces. In addition, the solution methods presented can be used for the characterization of different curve types. 


\section{Compliance with ethical standards}

\section{Acknowledgments}

This work was supported by the Scientific and Technological Research Council of Turkey (TÜBİTAK, The project numbered 119F213 titled Analysis of Constant Width Curves and Preliminary Study of the Scope of Application).

\section{Disclosure of conflict of interest}

There is no personal or financial conflict of interest for the authors of the article.

\section{References}

[1] Köse Ö. On space curves of constant breadth. Doğa Turk J Math. 1986; 10:11-14.

[2] Martini H, Montejano-Peimbert L, Oliveros D. Bodies of Constant Width: An Introduction to Convex Geometry with Applications. Springer Nature Switzerland AG; 2019.

[3] Euler L. De curvis trangularibis. Acta Academica Petropoliteanca. 1778- 1780.

[4] Reuleaux F. The Kinematics of Machinery. Trans By Kennedy ABW. New York: Dover Pub; 1963.

[5] Altunkaya B, Aksoyak FK. Curves of constant breadth according to Darboux frame. Commun Fac Sci Univ Ank Series A1. 2017; 66: 44-52.

[6] Ball NH. On Ovals. American Mathematical Monthly. 1930; 27:348-353.

[7] Mellish AP. Notes of differential geometry. Annals of Mathem. 1931; 32:181-190.

[8] Struik DJ. Differential geometry in the large. Bulletin Amer Mathem. 1931; 37:49-62.

[9] Zayas EE, Cardona S, Jordi L. Analysis and synthesis of the displacement function of the follower in constantbreadth cam mechanisms. Mechanism and Machine Theory. 2009; 44:1938-1949.

[10] Shchekotov M. Indoor localization methods based on Wi-Fi lateration and signal strength data collection, Conference of Open Innovation Association (Fruct). 2015; 186-191.

[11] Tripathi A, Gupta H, Dutta T, Mishra R, Shukla K, Jit S. Coverage and connectivity in WSNs: A Survey, research issues and challenges. IEEE Access. 2018; 6:26971-26992.

[12] Zhu C, Zheng C, Shu L, Han G, A survey on coverage and connectivity issues in wireless sensor networks. Journal Network and Computer Applications. 2012; 35:619-632.

[13] Kolaei A, Rakheja S, Richard MJ. Effects of tank cross-section on dynamic fluid slosh loads and roll stability of a partly-filled tank truck. European Journal of Mechanics B/Fluids. 2014; 46:46-58.

[14] Aydın TA, Sezer M. Hermite polynomial approach to determine spherical curves in Euclidean 3-space. New Trends in Math Sci. 2018; 6:189-199.

[15] Kocayiğit H, Önder M. Space curves of constant breadth in Minkowski 3-space. Annali di Matematica Pura ed Applicata, 2013; 192:805-814. 